\title{
Prevention of breast cancer skeletal metastases with parathyroid hormone
}

\author{
Srilatha Swami, ${ }^{1}$ Joshua Johnson, ${ }^{1,2,3}$ Lance A. Bettinson, ${ }^{1}$ Takaharu Kimura, ${ }^{1}$ Hui Zhu, ${ }^{1}$ \\ Megan A. Albertelli, ${ }^{4}$ Rachelle W. Johnson, ${ }^{2,3,5,6}$ and Joy $\mathrm{Y}$. $\mathbf{W u}^{1}$ \\ 'Department of Medicine, Division of Endocrinology, Stanford University School of Medicine, Stanford, California, USA. \\ 2Department of Medicine, Division of Clinical Pharmacology, and ${ }^{3}$ Vanderbilt Center for Bone Biology, Vanderbilt University \\ Medical Center, Vanderbilt University, Nashville, Tennessee, USA. ${ }^{4}$ Department of Comparative Medicine and ${ }^{5}$ Department \\ of Radiation Oncology, Division of Radiation and Cancer Biology, Stanford University School of Medicine, Stanford, \\ California, USA. ${ }^{6}$ Department of Cancer Biology, Vanderbilt University, Nashville, Tennessee, USA.
}

\begin{abstract}
Advanced breast cancer is frequently associated with skeletal metastases and accelerated bone loss. Recombinant parathyroid hormone [teriparatide, PTH(1-34)] is the first anabolic agent approved in the US for treatment of osteoporosis. While signaling through the PTH receptor in the osteoblast lineage regulates bone marrow hematopoietic niches, the effects of anabolic PTH on the skeletal metastatic niche are unknown. Here, we demonstrate, using orthotopic and intratibial models of 4T1 murine and MDA-MB-231 human breast cancer tumors, that anabolic PTH decreases both tumor engraftment and the incidence of spontaneous skeletal metastasis in mice. Microcomputed tomography and histomorphometric analyses revealed that PTH increases bone volume and reduces tumor engraftment and volume. Transwell migration assays with murine and human breast cancer cells revealed that PTH alters the gene expression profile of the metastatic niche, in particular VCAM-1, to inhibit recruitment of cancer cells. While PTH did not affect growth or migration of the primary tumor, it elicited several changes in the tumor gene expression profile resulting in a less metastatic phenotype. In conclusion, PTH treatment in mice alters the bone microenvironment, resulting in decreased cancer cell engraftment, reduced incidence of metastases, preservation of bone microarchitecture and prolonged survival.
\end{abstract}

Conflict of interest: The authors have declared that no conflict of interest exists.

Submitted: September 26, 2016 Accepted: July 27, 2017 Published: September 7, 2017

Reference information: JCI Insight. 2017;2(17):e90874. https://doi.org/10.1172/jci. insight. 90874

\section{Introduction}

Breast cancer is the second leading cause of death among women in the US, accounting for approximately $30 \%$ of new cancer diagnoses and $15 \%$ of cancer-related deaths (1). While early diagnosis and improved therapies have reduced the mortality rates associated with this disease, $20 \%$ develop distant metastases, which portend incurable disease (2). Bone is one of the most favored sites for breast cancer metastases, and serious skeletal-related events, including pathological fractures, spinal compression, and bone pain, are major causes of morbidity (3). The development of skeletal metastases depends on the interactions between the tumor cells and the bone microenvironment and involves many of the same pathways used to support normal hematopoietic stem cells (HSCs) (4-11). Among the best characterized are CXCL12/ CXCR4, Jag1/Notch, integrins, and TGF- $\beta$ (12-15). Thus, manipulation of the niche to make it less favorable to cancer cell homing is an important approach towards reducing breast cancer metastases.

Bone remodeling involves focal resorption by osteoclasts and new bone formation by osteoblasts. In women with breast cancer, systemic effects of the tumor, estrogen deprivation secondary to chemotherapy or aromatase inhibition, and glucocorticoid treatment all promote rapid bone loss (16-18). The current standard therapies for breast cancer-related bone metastases are antiresorptive medications, including bisphosphonates and denosumab, a monoclonal antibody targeted against the osteoclast differentiation factor RANKL $(19,20)$. Although these medications are effective in controlling adverse skeletal events, at high doses they are associated with increased risk of osteonecrosis of the jaw (21-23).

An alternative to antiresorptives is anabolic therapy targeted to promote bone formation. Studies have suggested a direct role for osteoblasts in the bone-tumor vicious cycle. Data from coculture experiments with multiple myeloma cells indicate that osteoblasts inhibit the growth of myeloma cells in bone (24). The addition of osteoblasts to cocultures of breast cancer cells and bone substrates decreased bone resorption 
by osteoclasts (25). Similarly, extensive bone loss due to breast cancer metastasis has been associated with the absence of osteoblasts (26). Hence, stimulation of osteoblast activity and new bone formation is an appealing therapeutic option for breast cancer patients.

Parathyroid hormone (PTH) and PTH-related peptide (PTHrP), both acting through the PTH receptor (PTH1R), play significant roles in bone remodeling and maintenance of calcium homeostasis (27). While the two ligands show very similar response profiles in activating various signaling pathways, the net effects of PTH or PTHrP on bone are dependent on the duration and exposure. For example, while intermittent administration of PTH or a PTHrP analog results in bone formation $(28,29)$, continual infusion of $\mathrm{PTH}$ or PTHrP results in increased resorption (30). Activation of the PTH1R receptor leads to regulation of multiple signaling pathways, including the PKA and PKC pathways. Recombinant PTH [teriparatide, $\mathrm{PTH}(1-34)$ ] administered intermittently (once daily) is the first anabolic treatment available for osteoporosis in the US $(29,31)$. Importantly, activation of PTH1R in osteoblasts in mice increases the numbers and engraftment of HSCs $(32,33)$. In postmenopausal women with osteoporosis, daily teriparatide treatment also increases circulating HSCs (34). Therefore, intermittent PTH is a useful therapeutic option to alter the bone microenvironment. In a murine model of multiple myeloma, PTH increased the expression of several osteoblastic markers, such as collagens and osteocalcin in bone (35). In mouse models of leukemia, activation of PTH1R in osteoblasts attenuated engraftment of chronic myelogenous leukemia while promoting that of acute myeloid leukemia (36), suggesting that the consequences of PTH signaling may differ by disease. However, the safety and efficacy of PTH in breast cancer is unknown.

In this study, we examined the effects of PTH administration on breast cancer skeletal metastases in mice. We hypothesize that intermittent PTH administration alters the bone microenvironment, rendering it less favorable for breast cancer cell colonization. We demonstrate for the first time to our knowledge that intermittent PTH reduces the incidence of breast cancer bone metastases in mice.

\section{Results}

Intermittent PTH decreases skeletal metastases and improves survival in metastatic breast cancer. To determine the effects of intermittent PTH on 4T1 mouse mammary tumor growth and metastasis, 6-week-old female BAL$\mathrm{B} / \mathrm{c}$ mice were pretreated with intermittent $\mathrm{PTH}(1-34)$ or vehicle (PBS) for 4 weeks (Figure 1A, pretreatment model). Microcomputed tomography $(\mu \mathrm{CT})$ analysis confirmed an increase in trabecular bone volume (Supplemental Figure 1, A-F; supplemental material available online with this article; https://doi.org/10.1172/ jci.insight.90874DS1) with PTH treatment. Orthotopic tumors were established by injection of 4T1-/LuBiG (4T1) cells into the mammary fat pad after 4 weeks, and PTH administration was continued for another 4 weeks. The mice were approximately 10 weeks of age at the time of tumor implantation. PTH is an important regulator of mineral metabolism. However, no changes were seen in serum calcium or $1,25(\mathrm{OH})_{2} \mathrm{D}$ levels with the dose of PTH used (Figure 1, B and C). No differences in the tumor volumes were detected between PTH pretreatment and PBS, with tumor volumes reaching approximately 1,500 $\mathrm{mm}^{3}$ at the end of 4 weeks (Figure 1D). Bioluminescence imaging (BLI) also revealed comparable tumor volumes in the two groups of mice (Figure 1E). Four weeks after tumor implantation, mice were euthanized and individual organs were imaged by BLI (Figure 2A). Metastases to lungs, liver, and spleen were similar in numbers and BLI intensity between the two groups (Figure 2). However, skeletal metastases to hind limbs were significantly decreased (7 of 20 mice) with PTH pretreatment as compared with 16 of 20 mice in the control group (Table 1). This was accompanied by a significant $(P<0.01)$ decrease in BLI signal intensity (Figure $2 \mathrm{~B}$ ), confirming a decrease in both incidence and intensity in skeletal metastasis with $\mathrm{PTH}$ pretreatment. Metastases to the hind limbs were also confirmed by histology, as seen in Supplemental Figure 1, G-J. Numbers of metastatic nodules did not differ in lungs, liver, or spleen with PTH pretreatment (Supplemental Figure 2, A-C). Likewise, no differences were seen by histological analyses (Supplemental Figure 2D).

We next delayed PTH until 24 hours after tumor implantation, with similar results (Figure 3A, treatment model). Mice were approximately 10 weeks of age at the time of tumor implantation. Four weeks after tumor implantation, the incidence and BLI intensity of skeletal metastases were significantly reduced with PTH treatment, with no differences in metastases to lungs, liver, or spleen (Figure 3, B and C, and Table 2). Double labeling by calcein injections revealed the presence of tumor cells in areas of active bone formation in both groups (Figure 3D). In a separate experiment, early invasion of cancer cells to bone was examined by flow cytometry for $\mathrm{GFP}^{+} 4 \mathrm{~T} 1$ cells in collagenase-digested bones and bone marrow of tumor-bearing mice administered PTH or PBS. No BLI signal was observed in hind limbs of either PTH- 
A

\section{PRE-TREATMENT MODEL}
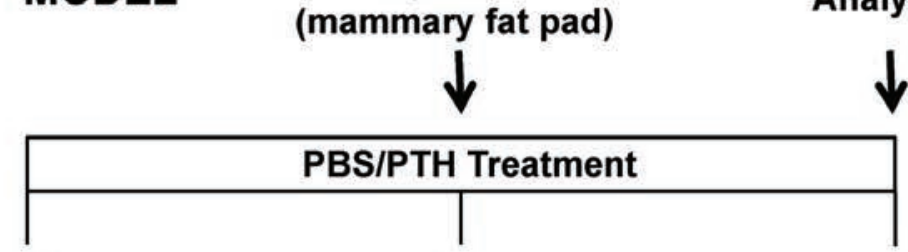

$-4$

C

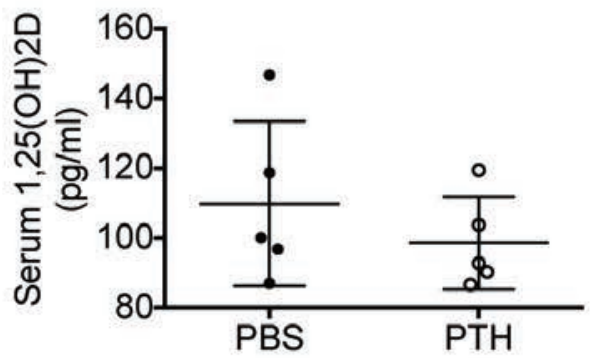

E

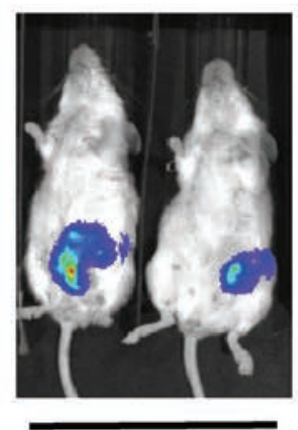

PBS
0
D

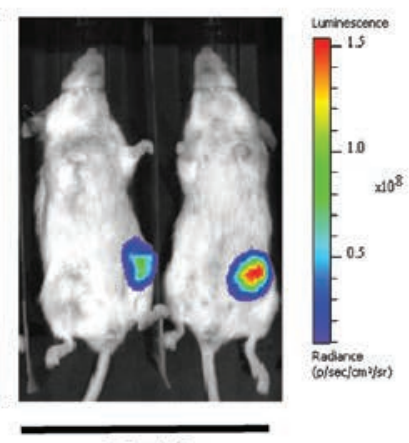

PTH 20 for each group.
B

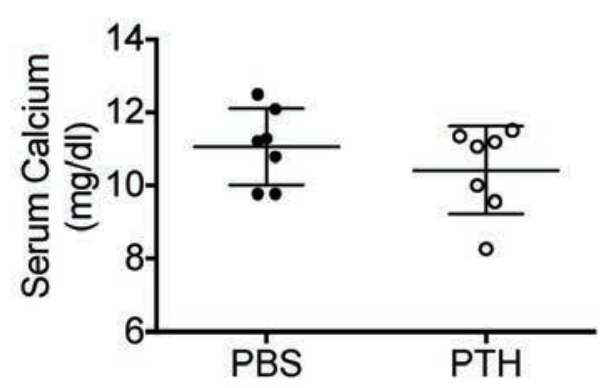

4 Week

Analysis
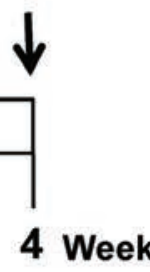

D

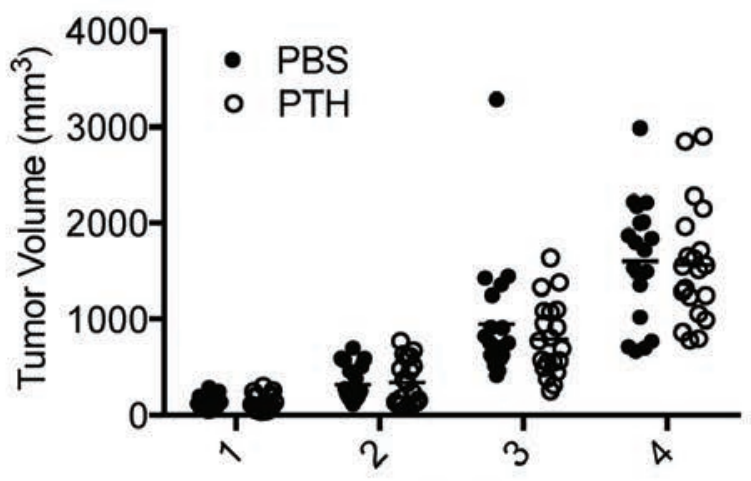

Weeks

Figure 1. Pretreatment with intermittent PTH does not affect primary tumor growth in an orthotopic 4T1 murine breast cancer model. (A) Prevention model experimental design. Mice were 6 weeks of age at the start of the experiment and 10 weeks at the time of $4 \mathrm{~T} 1$ cell injection. (B) Serum calcium and (C) serum $1,25(\mathrm{OH})_{2}$ D levels from PBS- and PTH-treated mice. (D) End point tumor volumes. (E) Representative bioluminescence images (BLI) of mice bearing 4T1 tumors pretreated with intermittent PTH. All values represent mean \pm SD of $n=$

or PBS-treated mice 3 weeks after tumor implantation (Figure 3E). However, flow cytometry revealed the presence of $\mathrm{GFP}^{+} 4 \mathrm{~T} 1$ cells in the PBS-treated control bones as early as week 1 (Supplemental Figure 3), while $\mathrm{GFP}^{+} 4 \mathrm{~T} 1$ cells were not detected in bones of the PTH-treated group until week 3 (Supplemental Figure 3 and Figure 3F). At week 3, 5 of 5 PBS-treated control bones showed varying percentages of GFP ${ }^{+}$ $4 \mathrm{~T} 1$ cells in contrast to only 2 of 5 bones in PTH-treated mice, confirming delayed engraftment of the cancer cells with PTH treatment (Figure 3F). Interestingly, under the conditions tested in this experiment, no $\mathrm{GFP}^{+}$cells could be detected in the bone marrow at week 3 (Figure $3 \mathrm{G}$ ) in either group.

To mimic the clinical setting where treatment would be initiated after diagnosis and debulking of primary tumors, orthotopic 4T1 tumors were allowed to grow for approximately 3 weeks before removal of the primary tumor (Figure 4A, survival model 1). Similar to the pretreatment and treatment models, mice were 10 weeks old at the time of tumor implantation. Tumor volumes were equivalent in both groups at the time of debulking (Supplemental Figure 4A). PTH/PBS treatment was started postoperatively and continued using survival as the end point. PTH increased median survival by $31 \%$ (25 days vs. 19 days in 
A

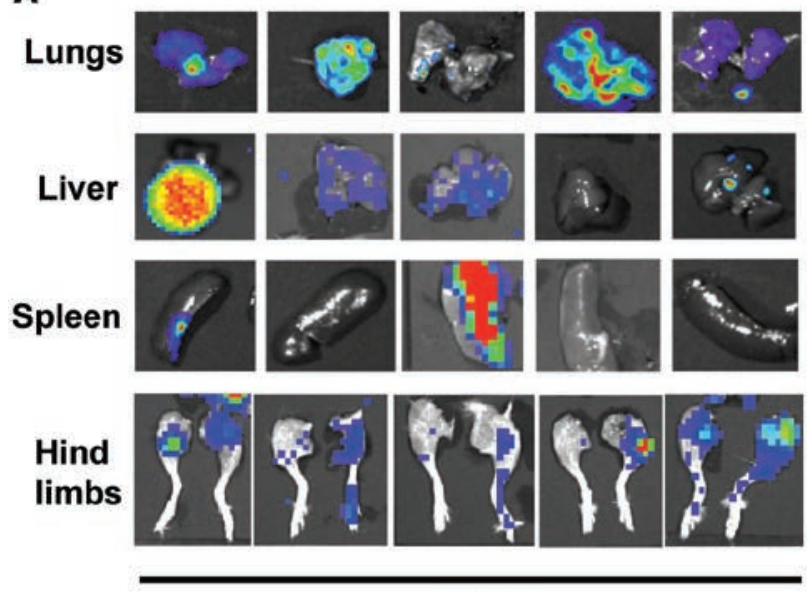

PBS
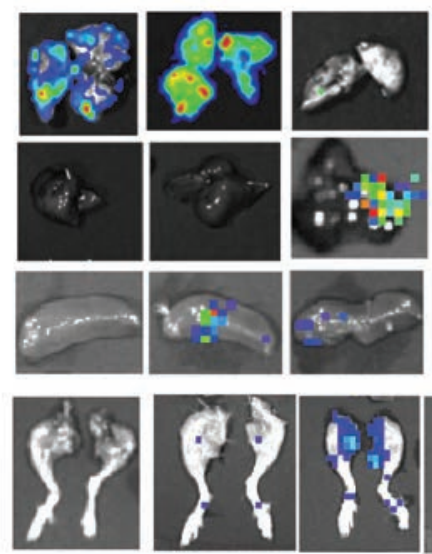

PTH
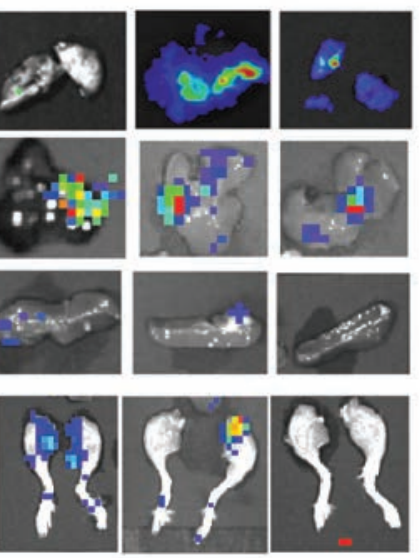

B

Lungs

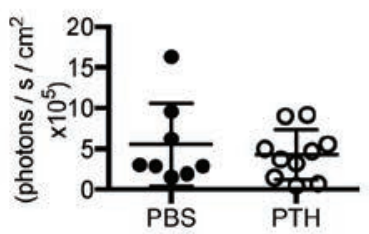

Liver

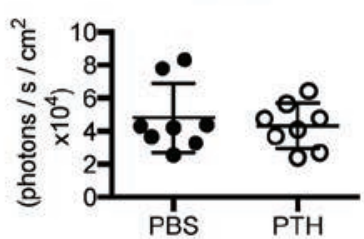

Spleen

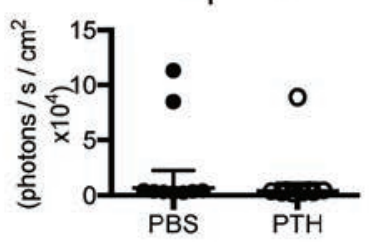

Hind limbs

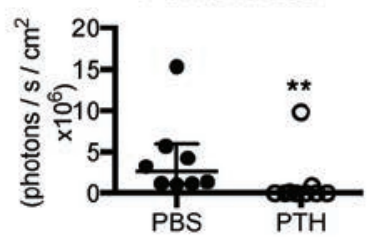

Figure 2. Pretreatment with intermittent PTH reduces skeletal metastases in an orthotopic 4T1 murine breast cancer model. (A) Representative BLI images of metastases to lungs, liver, spleen, and hind limbs in the pretreatment model. (B) Quantitation of BLI in lungs, liver, spleen, and hind limbs with metastases. All values represent mean $\pm S D$ of $n=10$ for each group. ${ }^{*} P<0.01$ when compared with PBS group, by 1-way ANOVA with Bonferroni's test as post-hoc analysis.

PBS-treated mice) (Figure 4B). In a second experiment, the primary tumors were removed approximately 2 weeks after 4T1 cell injection (Figure 4C, survival model 2). Tumor volumes were smaller than those in survival model 1, but they were similar in both groups at the time of debulking and they were histologically similar, as confirmed by H\&E staining (Supplemental Figure 4, B and C). Wound healing was similar in both groups 1 week after surgery, and no evidence of primary tumor was detected by BLI (Supplemental Figure 4D). Kaplan-Meier survival curves revealed improved survival in both groups with earlier debulking (Figure 4, B and D), and, even with earlier tumor removal, PTH increased median survival by $20 \%$ (36 days vs. 30 days in PBS-treated mice) (Figure 4D). 40\% of PTH-treated mice survived beyond the follow-up period of 6 weeks after surgery compared with $20 \%$ of PBS-treated control mice (Figure 4D). The primary cause of death in both groups was due to reappearance of primary tumors, followed by lung metastasis (Figure 4, E-G), with skeletal metastasis seen only in 1 mouse in the PBS-treated control group.

Overall, these results demonstrate that, in a model of spontaneous metastasis using 4T1 primary tumors, intermittent PTH substantially reduces skeletal metastases, without affecting primary tumor growth or metastases to other internal organs. Furthermore, even when delayed until after debulking of the primary tumor, PTH treatment yielded a small but significant increase in survival.

PTH reduces breast cancer cell engraftment in bone. PTH treatment may reduce breast cancer skeletal metastases through direct effects on the skeletal microenvironment and/or on the primary tumor as well as indirect effects on systemic mineral metabolism. To study the interactions between breast cancer cells and the bone microenvironment, 4T1 cells were directly injected into the intratibial bone marrow cavity of female mice pretreated with PTH/PBS for 4 weeks (Figure 5A). Baseline imaging and flow cytometry performed 6 hours after $4 \mathrm{~T} 1$ injections showed no differences in the number of GFP ${ }^{+} 4 \mathrm{~T} 1$ cells in the two groups of mice, confirming that seeding density was similar in both groups (Supplemental Figure 5). After 4 weeks, tumors were identified by BLI imaging in only 7 of 15 PTH-treated mice compared with 14 of 15 PBS-treated mice (Figure 5B). Additionally, the intensity of the BLI signal was also decreased signifi- 
Table 1. Numbers of metastases in lungs, liver, spleen, and hind limbs in the pretreatment model

\begin{tabular}{lccc}
\hline Model & & Pretreatment & \\
Group & PBS & PTH & $P$ value \\
Lungs & $19 / 20$ & $18 / 20$ & NS \\
Liver & $8 / 20$ & $6 / 20$ & NS \\
Spleen & $4 / 20$ & $5 / 20$ & NS \\
Hind limbs & $16 / 20$ & $7 / 20^{A}$ & $<0.001$
\end{tabular}

${ }^{A} P<0.001$ when compared with the PBS group, by Z proportion scores. cantly with PTH treatment (Figure 5C), suggesting that both engraftment and growth are reduced with $\mathrm{PTH}$ treatment.

Next, tibiae of mice pretreated with PTH or PBS were sham injected or injected with $4 \mathrm{~T} 1$ cells and evaluated by $\mu \mathrm{CT}$ and histology. As anticipated, PTH treatment significantly increased bone volume, as measured by $\mu \mathrm{CT}$ in the sham PTH tibiae when compared with the control (sham PBS) mice (Figure 5, D-F). 4T1 cells are known for their osteolytic activity. While PTH treatment preserved bone volume and trabecular thickness, even in the 4T1-injected tibiae, we observed a trend towards decreased trabecular bone volume in the 4T1-injected controls (4T1-PBS) (Figure 5D). End point evaluation of the tibiae, however, revealed marked osteolysis of bone microarchitecture in all 4T1-PBS mice (Figure $5 \mathrm{G}$ ) and a significant reduction in tumor burden in 4T1-PTH mice (Figure 5, G and H). The nonsignificant decrease in bone volume in the 4T1-PBS mice could be due an insufficient number of samples with sufficient trabecular bone available for $\mu \mathrm{CT}$ analysis due to tumor erosion. These results are in agreement with the BLI observations in which most of the control mice (Figure 5B) showed presence of tumor cells. Additionally, a greater number of tartrate-resistant acid phosphatase-positive (TRAP-positive) osteoclasts was found on the surfaces of both trabecular and cortical bone in the control mice when compared with PTH-treated mice (Figure 5, I and J).

To examine whether PTH has similar effects on human breast cancer cells, MDA-MB-231-fLuc (MDA) cells were injected into the tibiae of athymic nude mice pretreated with either PTH or PBS (Figure 6A). The results were similar to those observed with $4 \mathrm{~T} 1$ cells. PTH reduced tumor engraftment in the tibiae (Figure 6B). Likewise, there was a significant decrease in BLI intensity in tumors from the PTH-treated mice (Figure 6C), suggesting that PTH affects both the engraftment and growth of human breast cancer cells in the bone microenvironment.

These observations suggest that, in the intratibial model, which bypasses the initial stages in primary tumor metastasis, PTH administration retained its beneficial effect on tumor metastasis by increasing bone volume, decreasing TRAP-positive osteoclasts, and significantly reducing tumor engraftment and tumor burden of both murine and human breast cancer cells.

Intermittent PTH treatment attenuates the migratory potential of breast cancer cells by altering the metastatic gene expression profile in MC3T3-E1 cells. To investigate the chemoattractant effects of MC3T3-E1 preosteoblastic cells on breast cancer cell migration, MC3T3-E1 cells were treated with intermittent PTH (6 of 24 hours) or continuous PTH and cocultured with 4T1 or MDA breast cancer cells in a Transwell migration assay system. As shown in Figure 7, A-C, and Supplemental Figure 6, A and B, the presence of MC3T3-E1 cells in the Transwell chamber dramatically increased the migratory potential of the breast cancer cells as compared with wells lacking MC3T3-E1 cells. The migratory potential of both 4T1 and MDA cells did not change with continuous PTH treatment (Figure 7A). However, treatment with intermittent PTH significantly decreased the migratory potential of both 4T1 and MDA cells compared with PBS-treated controls (Figure 7B). Conditioned media from MC3T3-E1 cells treated with intermittent PTH also decreased the migratory potential of both the cell lines (Figure 7C), suggesting that inhibition of breast cancer cell migration is mediated at least in part by secreted factors. Rankl, Vcam-1, Vegfr1, Il7, and Opg are target genes known to play a critical role in the vicious cycle that drives the growth of breast cancer cells in the bone (37). In MC3T3-E1 cells treated with intermittent PTH, mRNA levels of these genes were significantly decreased (Figure 7D). Interestingly, although Opg expression did not change with PTH treatment, the Rankl/Opg ratio decreased significantly. These changes suggest an important role for intermittent PTH in altering the bone microenvironment.

To identify additional genes potentially regulated by PTH in osteoblasts, MC3T3-E1 cells treated with intermittent PTH were profiled using the RT ${ }^{2}$ Profiler Tumor Metastasis PCR Array from Qiagen. Of a total of 88 genes on the array, 26 genes were significantly downregulated and 5 genes were upregulated by PTH treatment (Figure 7E, Table 3, and Supplemental Table 1). Functional clustering revealed significant decreases in the expression of several matrix metallopeptidases (Mmp-10, Mmp-11, and Mmp-13), regulators of the bone-tumor 
A

TREATMENT MODEL

4T1-BGL cell

Injection

(mammary fat pad)

$\downarrow$

C
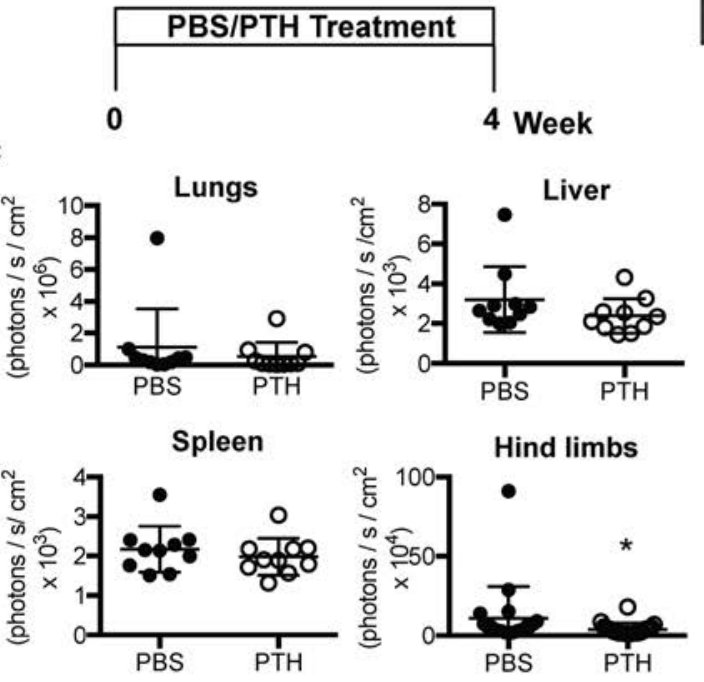

B
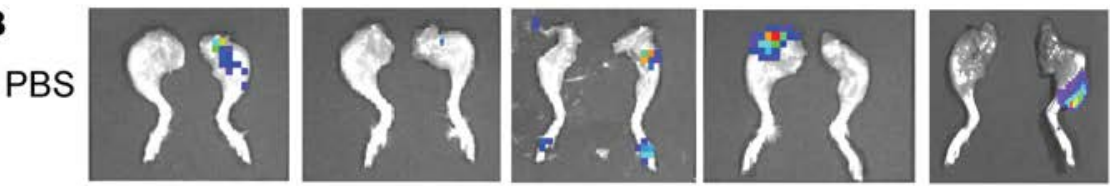

PTH
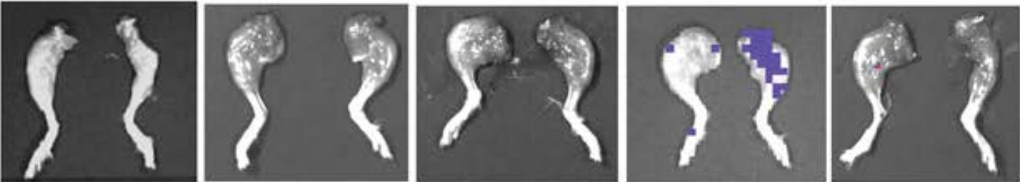

F Week 3 - Bone
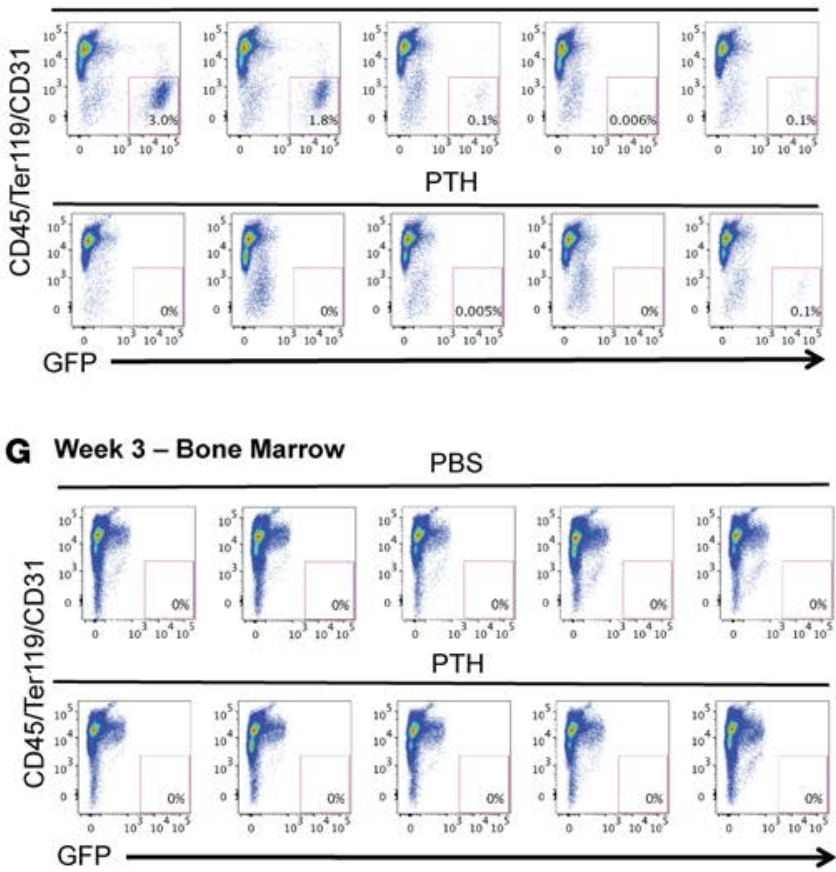

(+) Control

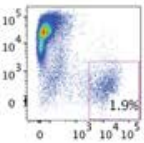

(+) Control

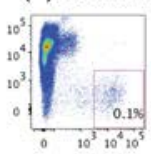


Figure 3. Intermittent PTH reduces skeletal metastases in a treatment model of 4T1 murine breast cancer. (A) Treatment model experimental design. Mice were 10 weeks old at the time of 4T1 cell injection (B) Representative BLI images of metastases to the hind limbs in the treatment model. (C) Quantitation of BLI in lungs, liver, spleen, and hind limbs with metastases in the treatment model. (D) Representative sections of double calcein labeling in hind limbs with metastases from mice treated with PBS/PTH. Sections are stained with xylene orange to visualize calcein labeling (indicated with white arrowheads) or H\&E to visualize corresponding histology (areas of tumor are indicated with dotted lines). Scale bar: $200 \mu \mathrm{m}$. (E) BLI at week 3 following PBS and PTH treatment in tumor-bearing mice $(n=5)$ in mice prior to flow cytometry. (F) Flow cytometry for detection of GFP+ 4 T1 cells in bones of PBSand PTH-treated mice $(n=5)$. In mice treated with PBS, GFP+ 4 T1 cells were detected in the hind limb bones of 1 of 5 animals at weeks 1 and 2 and in 5 of 5 mice at week 3. In PTH-treated mice, GFP+ 4 T1 cells were detected in 0 of 5 mice at weeks 1 and 2 and in 2 of 5 mice at week 3 . Hind limbs injected intratibially with $4 \mathrm{~T} 1$ cells were used as positive (+) controls. (C) Flow cytometry for detection of GFP+ 4 T1 cells in bone marrow $(n=5)$. No GFP+ 4 T1 cells were detected by flow cytometry in bone marrow from any of the 5 PBS- or PTH-treated mice at weeks 1,2 , or 3 . All values represent mean \pm SD. ${ }^{*} P<0.05$ when compared with PBS-treated group, by 1-way ANOVA with Bonferroni's test as post-hoc analysis.

vicious cycle. Likewise significant decreases were seen in the expression of Tgf $\beta$ and Cxcl-12, which are involved in the growth and migration of tumor cells to the bone. Interestingly, a significant decrease was noticed in the expression of cathepsin $K$, a gene predominantly associated with osteoclasts. Expression of the genes that were significantly altered by intermittent PTH was further validated using real-time PCR. With the exception of $C x c r 4$ and cathepsin $K$, all other genes showed a similar pattern of changes (Figure 7F). Since the $C x c r 4 / C x c l-12$ axis has been shown to play an important role in the homing of cancer cells to the bone, we confirmed by ELISA the changes in CXCL-12 expression. PTH decreased CXCL-12 levels in both the absence and presence of 4T1 cells (Figure 7G). Together, these experiments indicate that treatment of osteoblasts with intermittent PTH, which reduces migration of both human and murine breast cancer cells (Figure 7B), significantly alters the expression of several genes implicated in metastases, rendering the niche less favorable for the homing of cancer cells.

PTH decreases expression of VCAM-1 to inhibit migration of $4 T 1$ cells. VCAM-1 belongs to the Ig superfamily and is known to mediate leukocyte-endothelial cell adhesion and signal transduction (38); increased expression of VCAM-1 has been linked to the progression of several cancers (38-40). Previous studies from our laboratory have demonstrated that removal of the PTH1R in osteoprogenitor cells is associated with increased expression of VCAM-1, suggesting that PTH may negatively regulate VCAM-1 (41). Here, we demonstrate that the expression of VCAM-1 was significantly reduced with intermittent PTH treatment (Figure 7D and Figure 8A). Immunohistochemical analyses revealed reduced expression of VCAM-1 with PTH pretreatment in mice with intratibial 4T1 injection as compared with sham-injected mice or PBS-treated control mice (Figure 8A). While overexpression of Vcam1 in MC3T3-E1 cells led to increased 4T1 migration (Figure 8B and Supplemental Figure 6C), PTH treatment inhibited the constitutive expression of $V$ cam1 mRNA (Figure 8C) and attenuated the increased migration of 4T1 cells towards VCAM-1-overexpressing MC3T3-E1 cells (Figure 8B and Supplemental Figure 6C). Conversely, blocking endogenous VCAM-1 expression with a neutralizing antibody (41) reduced migration of 4T1 cells towards MC3T3-E1 cells, and PTH treatment had no additive effects (Figure 8D and Supplemental Figure 6D).

To further characterize the role of VCAM-1, mice were treated with VCAM-1-neutralizing antibody, IgG control, or PTH, as shown in Figure 8E. 4T1 cells were injected into the mammary fat pads of mice, and treatments were started 24 hours after the injection. After 4 weeks of treatment, the mice were euthanized for end point imaging by BLI (Figure 8F). Like the PTH-treated mice, anti-VCAM-1 treatment did not decrease metastases to lungs, liver, or spleen (Table 4). However, both PTH and anti-VCAM-1 treatment decreased metastases to the hind limbs (Table 4). These results suggest that VCAM-1 is an important mediator of 4T1 migration to the bone and that PTH regulates the expression levels of VCAM-1.

$P T H$ administration does not affect $4 T 1$ cell growth in vitro or tumor progression in $B A L B / c$ mice. To assess the direct effects of PTH administration on breast cancer cells and primary tumors, 4T1 cells were treated with graded concentrations of intermittent PTH. There were no differences in proliferation between the vehicle and PTH-treated cells, as measured by DNA content (Figure 9A), and intermittent PTH treatment did not change the migratory potential on 4T1 cells in a Transwell assay (Figure 9B). Similarly no differences were seen in tumor volumes between the PBS-treated control mice and PTH-treated mice, either with PTH treatment (Figure 9, C and D) or pretreatment (Figure 1, D and E). However, analysis of select genes involved in tumor growth and metastasis revealed that intermittent $\mathrm{PTH}$ pretreatment attenuated the expression of several key genes (Pthrp, Cxcr4, Cxcr7, Tnfa, and Ilo) in 4T1 primary tumors (Figure 9E). While expression of Cxcr7 mRNA was decreased with PTH treatment, no differences could be seen at the protein level (Figure 9F). Of note, mRNA levels of $p 21$, a classic marker for cell cycle arrest, were unaffected by PTH treatment, suggesting that the effects of $\mathrm{PTH}$ are not mediated through growth arrest. 
Table 2. Numbers of metastases in lungs, liver, spleen, and hind limbs in the treatment model

\begin{tabular}{lccc}
\hline Model & & Treatment & Pvalue \\
Group & PBS & PTH & NS \\
Lungs & $16 / 20$ & $15 / 20$ & NS \\
Liver & $1 / 20$ & $0 / 20$ & NS \\
Spleen & $1 / 20$ & $0 / 20$ & $<0.05$ \\
Hind limbs & $10 / 20$ & $5 / 20^{A}$ & \\
AP $<.05$ when compared with the PBS-treated group, by Z proportion scores. & \\
\hline
\end{tabular}

\section{Discussion}

Bone is a favored site for breast cancer metastasis, and the development of skeletal metastases depends on the interactions between the tumor cells and the bone metastatic niche. Antiresorptive therapies, including bisphosphonates and denosumab, are effective in reducing adverse skeletal events associated with breast cancer $(42,43)$. Breast cancer patients are also at risk for osteoporosis and fragility fractures. Teriparatide [recombinant PTH(1-34)] is the first FDA-approved anabolic drug available to treat osteoporosis in the US. Caution has been associated with the use of this drug due to the development of rodent osteosarcoma in preclinical testing (44-46). However, in over 10 years (approved in November 2002) on the market, there have been only three cases of osteosarcoma in humans treated with teriparatide, suggesting that the incidence of osteosarcoma in patients taking teriparatide is not higher than that observed in the general population $(47,48)$. The safety and efficacy of anabolic therapies in breast cancer is unknown.

It is well established that PTH stimulates both bone formation and resorption, and the dynamic balance is dependent on the dose and kinetics of the PTH signal. While continuous PTH exposure results in active bone resorption, intermittent PTH exposure favors bone formation $(49,50)$. At intermittent doses, PTH binds to the PTH1R and transiently regulates mRNAs encoding for transcription factors, cytokines, and growth factors via the cAMP/PKA signaling pathways, leading to an overall anabolic effect $(49,51)$. In contrast, the OPG/RANKL/RANK pathway appears to be the prime mediator for the actions of continuous PTH ( 50 , 52). A DNA microarray study (53) of gene expression changes in rats treated with continuous or intermittent PTH for 7 days observed that both treatments resulted in gene expression patterns that matched the anticipated histomorphometric changes. Continuous PTH treatment upregulated 173 unique genes, many of which were associated with increased osteoclast number and bone resorption (e.g., MMPs and cathepsin K), as opposed to intermittent PTH treatment, in which only 19 genes were uniquely regulated, many of which were associated with bone formation. A more comprehensive study conducted by Li et al. (54) also showed similar findings, that intermittent PTH was anabolic and continuous PTH was catabolic in its effects on rat bones. In humans, the catabolic effects of PTH are best represented by hyperparathyroidism (HPT), a classic disorder that is associated with excess circulating PTH, hypercalcemia, increased circulating 1,25 $(\mathrm{OH}) 2 \mathrm{D}$ levels (55), and a net catabolic effect resulting in bone loss. Several existing studies suggest a potential association between HPT and breast cancer. Among women who underwent surgery for primary parathyroid adenoma in Sweden, the number of incident breast cancer cases was slightly greater than expected (56, 57). Conversely, the prevalence of HPT among women with breast cancer may also be increased (57-59). However, the safety of teriparatide in breast cancer and skeletal metastases has not been examined.

In our study, we used the anabolic or intermittent dose of PTH to study the effects on the bone microenvironment in mice bearing primary breast tumors. Previous studies with anabolic PTH reported that PTH increased the expression of several osteoblastic markers, such as collagens and osteocalcin in bone, in a murine model of multiple myeloma (35). In mouse models of leukemia, however, activation of PTH1R in osteoblasts attenuated engraftment of chronic myelogenous leukemia while promoting that of acute myeloid leukemia (36). In another study (60), it was shown that increasing the number of osteoblastic lineage cells with anabolic PTH prior to inoculation of prostate cancer increased the number of cancer cells in bone marrow, suggesting that prostate tumor growth in bone was dependent on the osteoblastic niches. Similarly, Schneider et al. (61) have shown that intermittent PTH treatment was associated with increased bone turnover and marrow cell proliferation, leading to increased prostate cancer tumor engraftment in the skeleton. Although these experimental designs differed slightly from the model used in our study, they support the hypothesis that the effects of PTH on metastatic niches may depend in part on the primary 
A

SURVIVAL MODEL 1

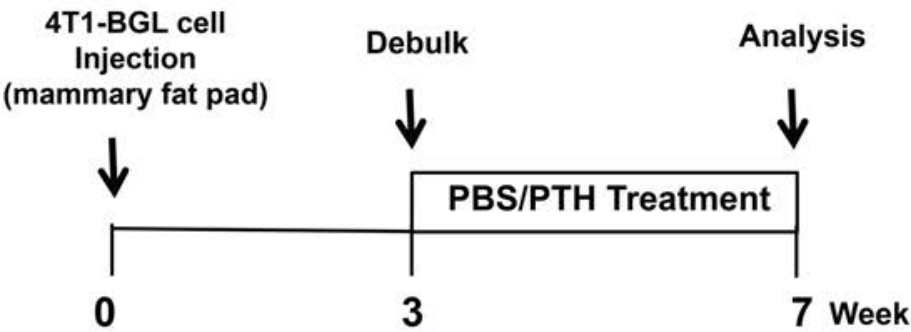

B

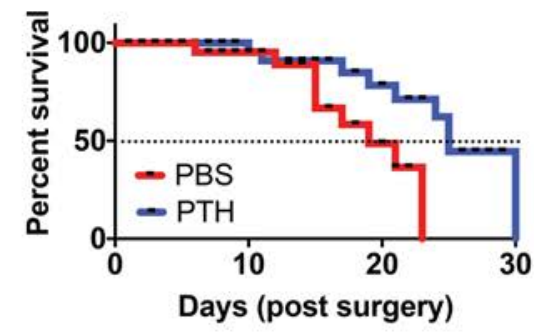

D

C

SURVIVAL MODEL 2

4T1-BGL cell

Injection (mammary fat

pad)

$\downarrow$

Debulk

Analysis

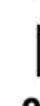

E

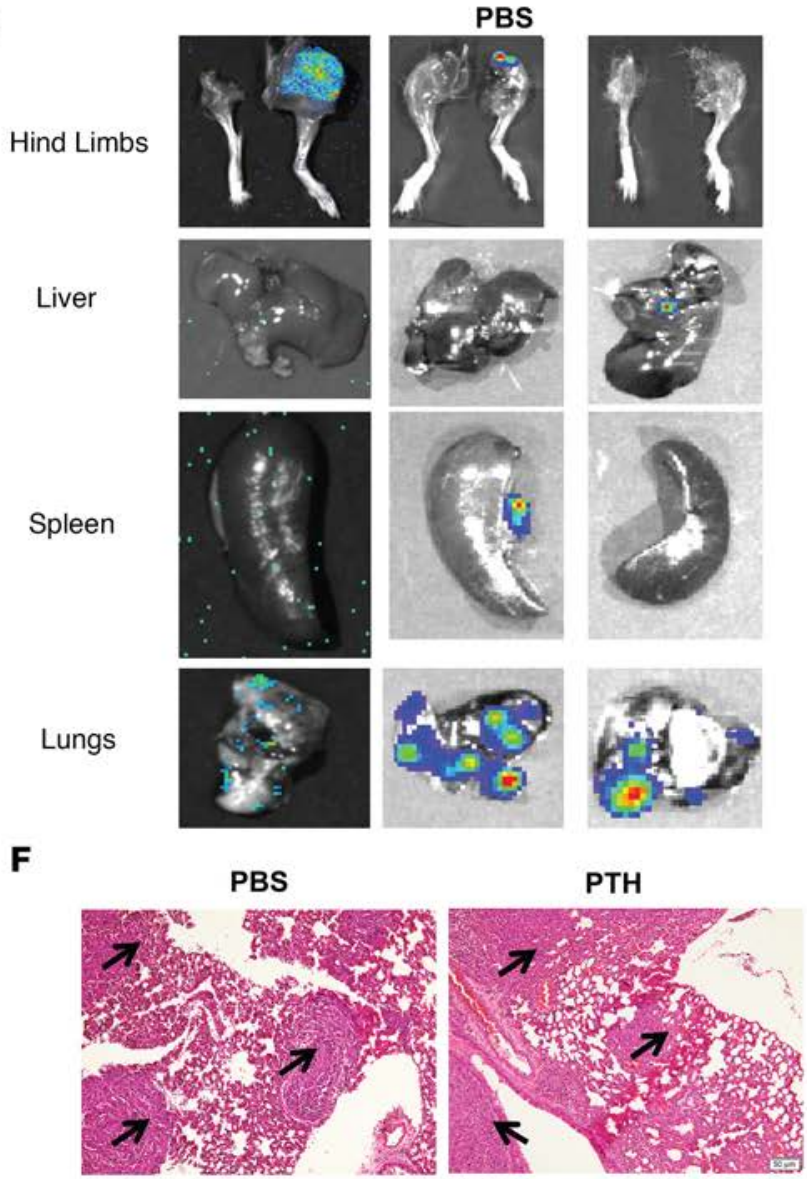

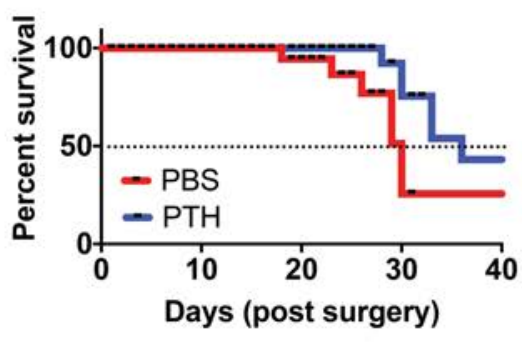

8 Week
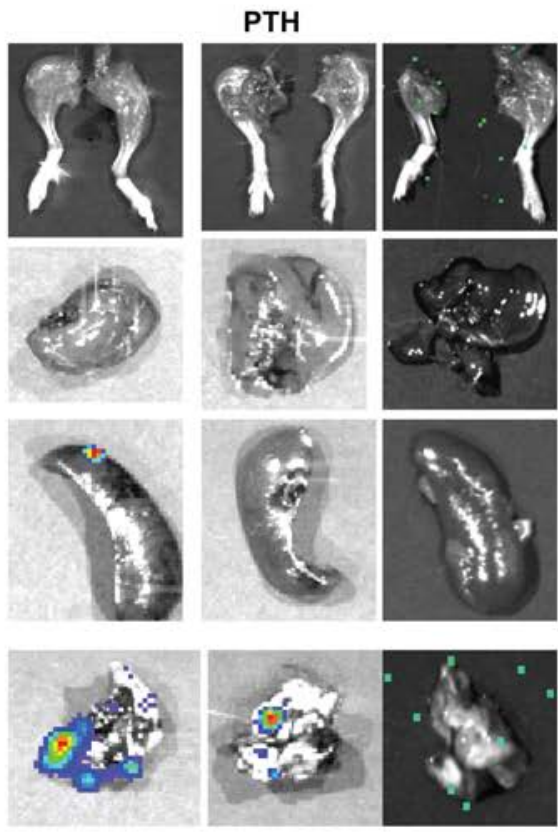

G

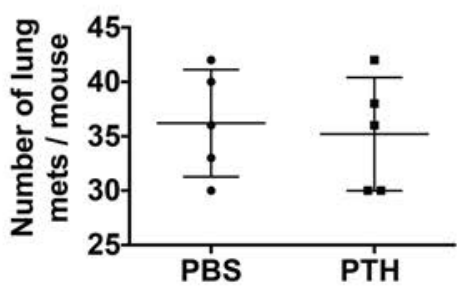


Figure 4. Treatment with intermittent PTH prolongs survival in mice undergoing tumor debulking surgery. (A) Survival model 1 experimental design ( $n$ $=10$ ). Mice were 10 weeks old at the time of tumor cell injections (B) Kaplan-Meier plots to assess survival after debulking of primary tumors in model 1 . Median survival of PBS-treated mice was 19 days and that of PTH-treated mice was 25 days $(P=0.0183$, log-rank [Mantel-Cox] test). (C) Survival model 2 experimental design $(n=10)$. (D) Kaplan-Meier plots to assess survival after debulking of primary tumors in model 2. Median survival of PBS-treated mice was 30 days and that of PTH-treated mice was 36 days $(P=0.029$, log-rank [Mantel-Cox] test). (E) Representative BLI imaging of skeletal and other distal organs at end point. (F) H\&E staining (areas of tumor are indicated with arrows) and (G) macroscopic examination of lungs at end point from mice in survival model 2 at end point. Scale bar: $200 \mu \mathrm{m}$. All values represent mean \pm SD for each group.

tumor type. In our study, we demonstrate presence of tumor cells in areas of active bone formation in both PBS-treated controls as well as PTH-treated mice. This is not surprising, since these areas are likely to be rich in growth factors, cytokines, and other factors that contribute to the tumor microenvironment. We show, for the first time to our knowledge, that administration of PTH reduces spontaneous breast cancer skeletal metastases and prolongs survival in mice.

Metastasis is a multistep sequential process involving proliferation and dissemination of the cancer cells from the primary tumor, intravasation and migration into circulation, extravasation into the bone, and finally colonization in specific niches (37). It is well established that the bone marrow has specific microenvironments that are conducive for disseminating cancer cells to engraft. The cancer cells compete for the same endosteal niche, which is rich in osteoblasts that are essential for normal hematopoiesis in adult bone. Since PTH acts on PTH1R on osteoblasts, PTH is likely to have a direct role on the bone marrow microenvironment, in which cells of the bone marrow and other stromal cells are involved in the creation of a premetastatic niche. Indeed our results demonstrate that PTH reduced tumor burden and colonization of bone by breast cancer cells directly injected into the intratibial bone marrow cavity, thus bypassing the initial steps in metastasis. PTH decreased breast cancer metastases to bone at least in part by altering VCAM1 expression. We have previously demonstrated increased expression of VCAM-1 with PTH1R ablation in osteoprogenitor cells (41), suggesting that PTH may be a negative regulator of VCAM-1 expression. Here, we report that PTH treatment reduced VCAM-1 expression and attenuated the ability of VCAM-1 to enhance breast cancer cell migration in vitro and in vivo.

PTH also significantly reduced the expression of other premetastatic niche-promoting factors, including MMPs (Mmp10, Mmp11, and Mmp13), TgfB, Rankl, and Vegfr1 (62-64). That Rankl is suppressed by PTH is somewhat surprising, given reports that PTH increases Rankl expression in stromal cells and osteoblasts $(65,66)$. These effects were, however, attributed to treatments with continuous PTH and not intermittent $\mathrm{PTH}$. In this study, we report that intermittent PTH treatment decreases Rankl expression in cocultures of osteoblasts with breast cancer cells, leading to a significant decrease in the RANKL/OPG ratio. This is an important finding, and it may reflect the altered microenvironment in the setting of bone metastases. These global gene changes with PTH likely result in a microenvironment that is less favorable to the homing of cancer cells. Furthermore, we have found that intermittent PTH decreased Cxcl12 gene expression and protein in MC3T3-E1 osteoblasts, with reciprocal decreases in Cxcr4 and Cxcr7 mRNA seen in the 4T1 primary tumors. In the normal bone marrow microenvironment, CXCL12 plays a significant role in the homing of circulating HSCs to the bone marrow niche, and cancer cells are known to hijack the CXCR4/CXCL12 axis to colonize the bone, thus competing with HSCs for the same niche (60). Decreasing the expression of both the ligand $(\mathrm{C} x \mathrm{cl} 12)$ and the receptor $(\mathrm{Cxcr} 4$ and $\mathrm{Cxcr}$ ) may be one of the key mechanisms for reduced engraftment of cancer cells to the bone with PTH treatment.

As for potential direct effects of PTH on the primary tumor, using both in vitro and in vivo models, we showed that PTH administration did not affect the proliferation of the $4 \mathrm{~T} 1$ breast cancer cells. These observations are consistent with our findings that only metastases to bone and not to other organs are reduced. However, PTH significantly decreased the expression of several genes in 4T1 primary tumors, including Il6 and Tnfa, important players in the bone-tumor vicious cycle that are implicated in the recruitment of leukocytes and endothelial cells into the tumor microenvironment to initiate matrix remodeling, an essential step for the dissociation of tumor cells from their primary tumor.

An interesting observation in this study was the decreased expression of Pthrp in the primary tumors with PTH treatment. PTHrP expression is commonly found in many types of cancers, including the breast, and it can promote tumor growth and metastasis $(67,68)$. While PTH and PTHrP have similar binding affinities to the G protein-coupled PTH1R, PTH binds with greater stability than the PTHrP, facilitating prolonged signaling even after internalization of the ligand-receptor complex, resulting in different downstream effects (69). Acting 

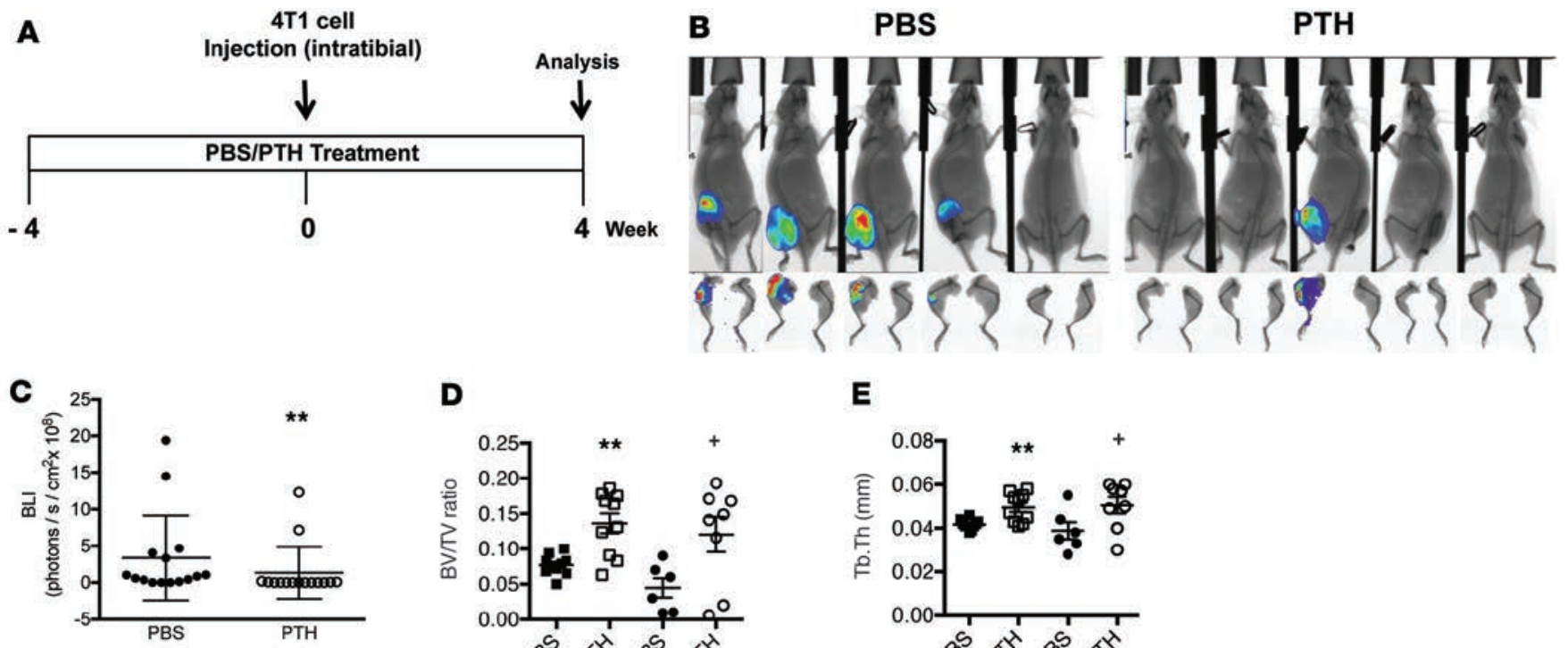

D

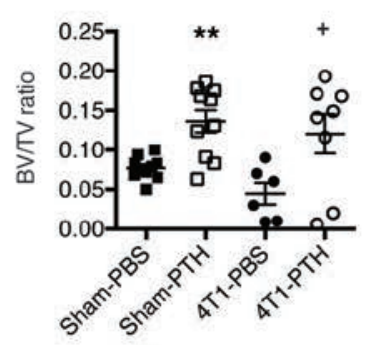

E

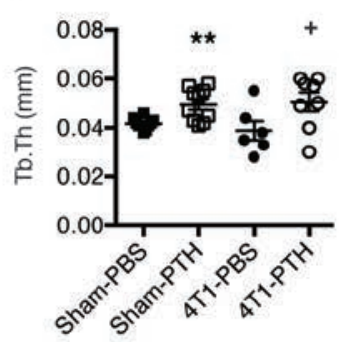

F

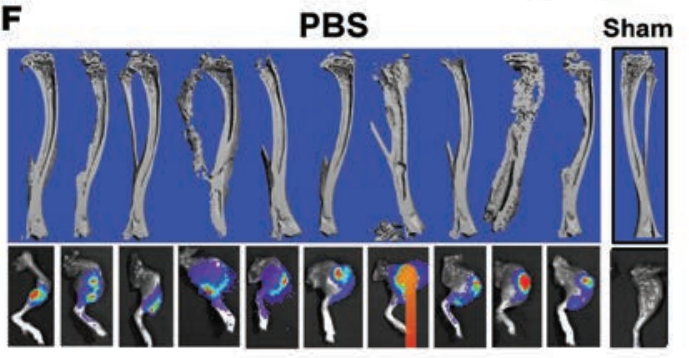

PTH

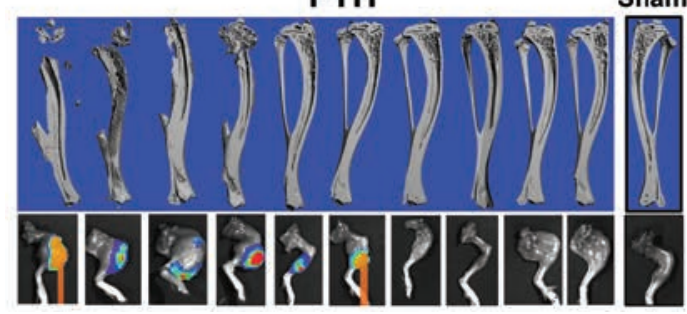

G

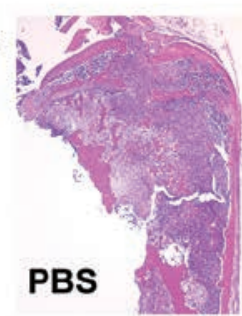

Trabecular

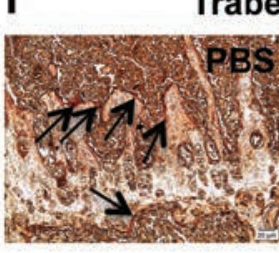

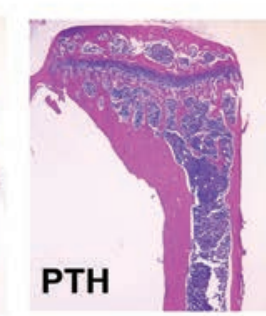

H

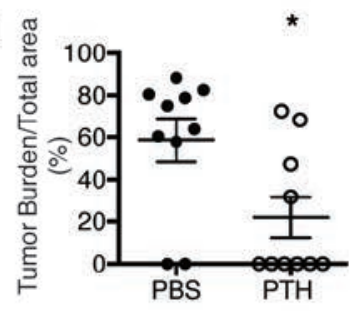

Figure 5. Intermittent PTH decreases 4T1 mouse breast cancer cell engraftment to the bone, leading to better preservation of bone architecture. (A) Intratibial injection experimental design. Mice were 6 weeks of age at the start of the experiment and 10 weeks at the time of 4 T1 cell injection. (B) Representative end point BLI images of hind limbs injected with $4 \mathrm{T1}$ murine breast cancer cells. After 4 weeks, tumors were identified by BLI imaging in 7 of 15 PTH-treated mice compared with 14 of 15 in the PBS-treated mice $(P=0.005)$. (C) Quantitation of BLI 4 weeks after intratibial injections of 4 T1 cells. Architectural analyses of proximal tibia (D) bone volume/total volume (BV/TV) and (E) trabecular thickness (Tb.Th) by $\mu$ CT $(n=10)$. $(\mathbf{F})$ Representative reconstructed 3D $\mu \mathrm{CT}$ images of tibiae with corresponding BLI images at end point in PBS-and PTH-treated mice following intratibial injections of 4T1 cells. (C) Representative H\&E-stained images of tibia from PBS- and PTH-treated mice with intratibial injections of 4T1 cells (original magnification, $\times 4$ ). (H) Quantitation of tumor burden. Representative TRAP staining of (I) trabecular region (original magnification, $\times 20$ ) and (J) cortical region (original magnification, $\times 10$ ) from tibiae of mice treated with PBS/PTH with intratibial injections of 4 T1 cells. TRAP-positive osteoclasts are indicated by arrows. All values represent mean \pm SD of $n=10$ for each group. ${ }^{*} P<0.05$, ${ }^{* *} P<0.01$ when compared with PBS group, by 1 -way ANOVA with Bonferroni's test as post-hoc analysis.

in a paracrine manner in the bone microenvironment, PTHrP activates osteoblasts and, in turn, osteoclasts to drive bone resorption. As a consequence, many other mitogenic factors are released from the bone matrix that further fuel the vicious cycle of tumor growth and bone resorption. Early investigations by Guise et al. revealed that tumor-produced PTHrP promotes breast cancer metastasis to the bone and neutralizing antibodies against PTHrP may reduce the development of osteolytic lesions as well as retard tumor growth in the bone (70). In our studies, we report reduced expression of Pthrp in the 4T1 tumors with intermittent PTH treatment. We also report decreased expression of $T g f \beta$ in the bone microenvironment. It has been shown that the TGF- $\beta$ released 


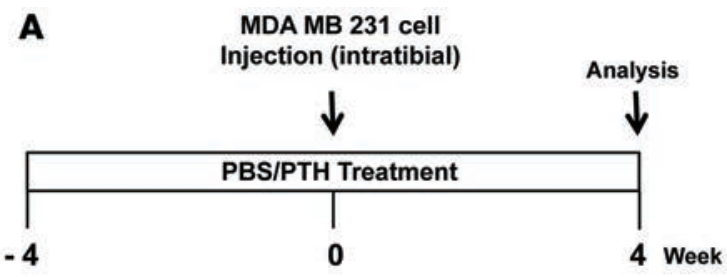

B

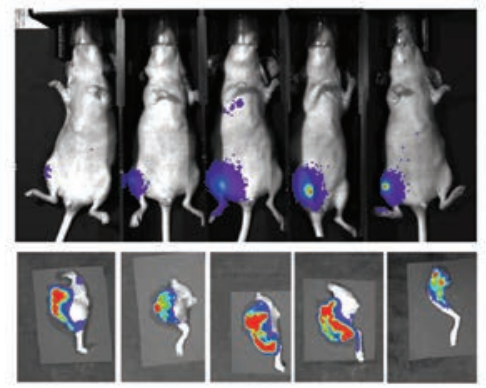

PTH

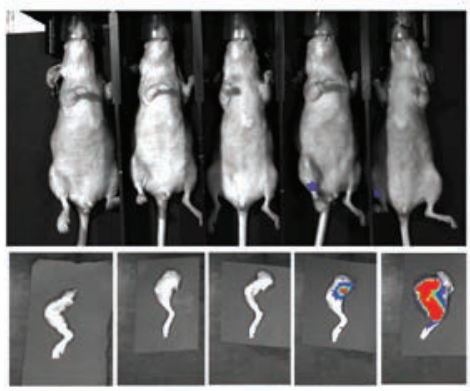

Figure 6. Pretreatment with intermittent PTH reduces MDA-MB-231 human breast cancer cell engraftment in bone. (A) Intratibial injection experimental design. Mice were 6 weeks of age at the start of the experiment and 10 weeks at the time of 4T1 cell injection.

(B) Representative end point BLI images of hind limbs injected with MDA-MB-231 human breast cancer cells. After 4 weeks, tumors were identified in 5 of 15 PTH-treated mice compared with 11 of 15 PBS-treated mice ( $P=0.027$, by 1 -way ANOVA with Bonferroni's test as post-hoc analysis). (C) Quantitation of BLI 4 weeks after intratibial injections of MDA cells. All values represent mean $\pm S D$ of $n=15$ for each group. ${ }^{*} P<0.05$, when compared with PBS group, by 1-way ANOVA with Bonferroni's test as post-hoc analysis.

due to tumor-stimulated bone resorption may drive the growth of breast cancer cells in the bone and that tumor PTHrP may be the effector for the actions of TGF- $\beta$ in the bone (71). Therefore, it is possible that decreased expression of Pthrp and TgfB inhibits the vicious cycle and contributes to a less attractive microenvironment for tumor cell growth and proliferation. Decreasing the expression of these key genes may contribute to the reduced migratory potential of the cancer cells, as observed in our Transwell migration assays with both 4T1 and MDA cells. Parallel to this, in an in vivo setting, we demonstrated that PTH administration and pretreatment not only changed the gene expression profile in the primary tumor microenvironment, but significantly reduced spontaneous metastases in orthotopic models of 4T1 primary tumors to the bone.

In conclusion, our studies demonstrate that anabolic PTH decreased both the tumor engraftment rate and the incidence of spontaneous breast cancer metastases in orthotopic models of 4T1 murine breast cancer tumors and prolonged survival in mice. In vitro experiments with human and murine breast cancer cells suggest that the molecular mechanisms involve alterations of the gene expression profile of the metastatic niche to render it less favorable to breast cancer cells. Although PTH had no effect on the growth of the primary tumor, the gene expression profile reflected a less metastatic phenotype. Our observations support the hypothesis that the effects of PTH on the bone microenvironment negatively affect breast cancer metastases to the bone. Anabolic PTH is an FDA-approved drug and a useful therapeutic option that clearly warrants further evaluation in preventing breast cancer metastasis.

\section{Methods}

\section{Breast cancer cells}

Murine 4T1 and human MDA-MB-231 breast cancer cells obtained originally from ATCC were genetically engineered to stably express firefly luciferase and enhanced green fluorescence protein using the Sleeping beauty transposon plasmid pKT2/LuBiG $(72,73)$. These cells are referred to as MDA (a gift from Bonnie King, Stanford University) and 4T1 (a gift from Michael Bachmann, Stanford University). The MDA 

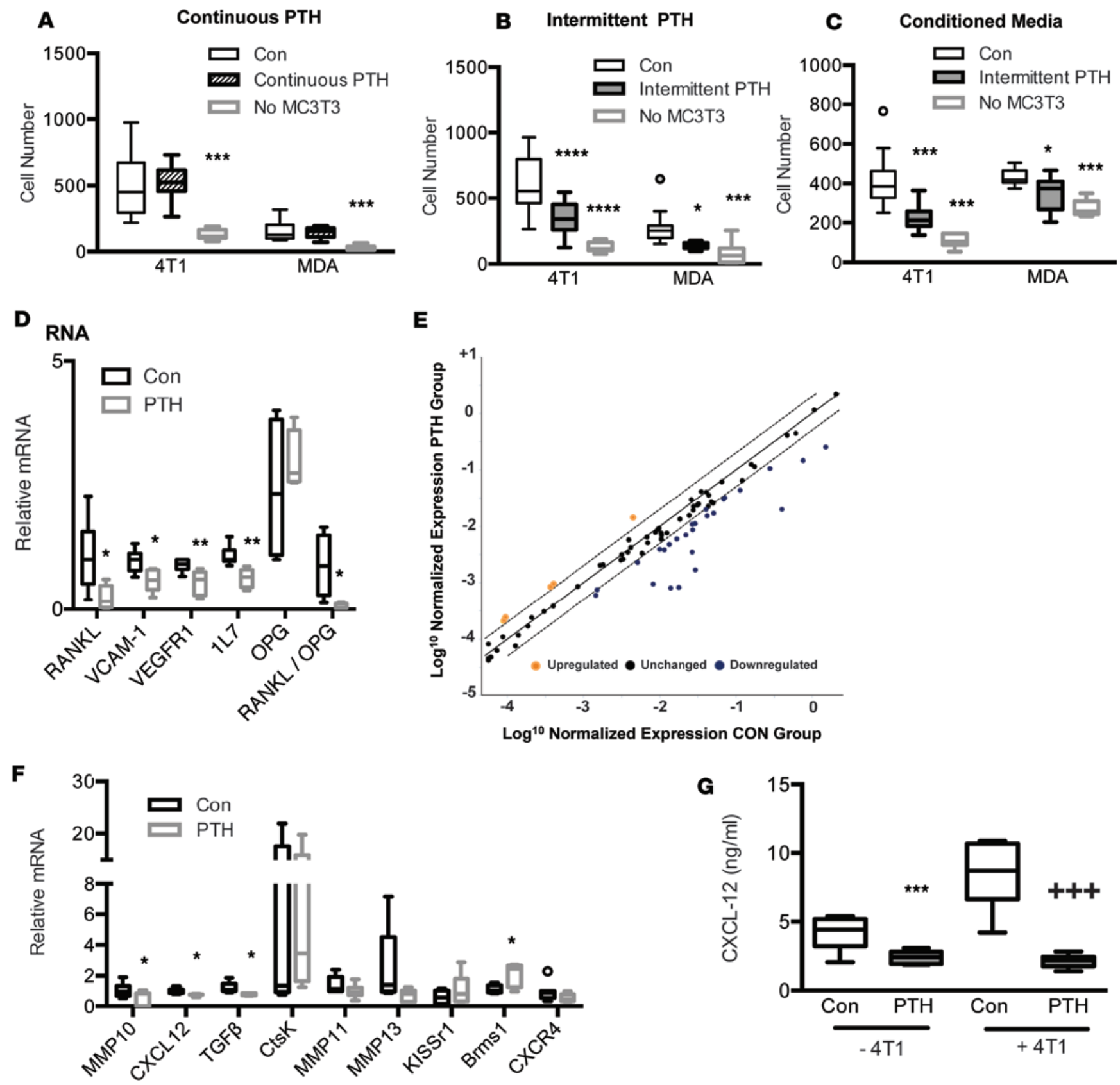

Figure 7. In vitro treatment with intermittent PTH attenuates the migratory potential of breast cancer cells by altering the gene expression profile in preosteoblastic MC3T3-E1 cells. Numbers of breast cancer cells (4T1 and MDA) that migrated towards MC3T3-E1 preosteoblastic cells treated with (A) continuous PTH, (B) intermittent PTH, or (C) conditioned media from cells treated with intermittent PTH in a Transwell migration system. (D) Target gene expression in MC3T3-E1 cells using gene-specific primers. (E) RT2 Profiler Tumor PCR Metastasis PCR Array analysis of MC3T3-E1 gene expression. (F) Genes with $>2$ fold changes from Table 3 were further validated with real-time quantitative PCR. (G) CXCL12 protein levels in MC3T3-E1 cells treated with control or PTH in the absence or presence of $4 \mathrm{~T} 1$ cells. All values represent mean $\pm \mathrm{SEM}$ of at least 3 individual experiments conducted in triplicate. ${ }^{*} P<$ 0.05 , ${ }^{* *} P<0.01,{ }^{* *} P<0.001$, ${ }^{* * *} P<0.0001$ when compared with control (con) group. ${ }^{++} P<0.001$ when compared with control (+4T1), by 1 -way ANOVA with Bonferroni's test as post-hoc analysis.

cells were authenticated by Genetica DNA Laboratories. In the absence of a universally accepted standard method for authentication of mouse cell lines, the 4T1 cells were also submitted to Genetica and were validated to be free of any cross contamination from human DNA. Cells were cultured in DMEM media supplemented with $10 \% \mathrm{FBS}, 100 \mathrm{IU} / \mathrm{ml}$ penicillin, and $100 \mu \mathrm{g} / \mathrm{ml}$ streptomycin and maintained at $37^{\circ} \mathrm{C}$ with $5 \% \mathrm{CO}_{2}$ in a humidified incubator. 
Table 3. Significant fold change in genes with PTH treatment

\begin{tabular}{|c|c|}
\hline Genes & Fold change \\
\hline \multicolumn{2}{|l|}{ Upregulated } \\
\hline Brms1 (breast cancer metastasis suppressor 1) & $3.2^{\mathrm{A}}$ \\
\hline \multicolumn{2}{|l|}{ Downregulated } \\
\hline MMP10 (matrix metallopeptidase 10) & $-21.39^{A}$ \\
\hline CXCL12 (chemokine [C-X-C motif] ligand 12) & $-20.08^{A}$ \\
\hline TGF $\beta 1$ (transforming growth factor $\beta 1$ ) & $-17.44^{A}$ \\
\hline Ctsk (cathepsin K) & $-17.14^{A}$ \\
\hline MMP11 (matrix metallopeptidase 11) & $-8.6^{A}$ \\
\hline MMP12 (matrix metallopeptidase 12) & $-7.71^{A}$ \\
\hline \multicolumn{2}{|c|}{${ }^{A} P<0.05$, by $1-w a y$ ANOVA with Bonferroni's test as post-hoc analysis. } \\
\hline
\end{tabular}

\section{Animal studies}

Mice. 10-week-old female BALB/c mice and athymic nude mice purchased from The Jackson Laboratory were housed in a designated pathogen-free area facility and fed irradiated mouse chow and autoclaved water. For the pretreatment model, pretreatment was started at 6 weeks of age and continued for 4 weeks. All models receive tumor cell injections at approximately 10 weeks of age.

Mammary fat pad injections. Orthotopic xenografts were established in approximately 10-week-old mice by injecting 4T1 cells ( $\sim 150,000$ cells $)$, suspended in $50 \mu 1$ culture medium/Matrigel mixture, directly into the fourth mammary fat pad on the left side. Body weights and tumor volumes were measured weekly, and tumor volumes were calculated from two tumor diameter measurements using the following formula: tumor volume $=$ (length $\times$ width $\left.^{2}\right) / 2(74)$.

Experimental design. Three different experimental approaches were used to assess the effects of anabolic PTH on primary tumor metastasis to distal organs.

In the pretreatment model (Figure 1A), 6-week-old mice were pretreated for 4 weeks with either intermittent PTH $[\mathrm{PTH}(1-34) ; 80 \mu \mathrm{g} / \mathrm{kg} / \mathrm{d}$, Bachem] or PBS. 4T1 cells were injected into the mammary fat pad to establish tumors, and then PTH (or PBS) was continued for another 4 weeks before terminal euthanasia. Individual organs collected and processed for BLI were fixed in $10 \%$ formalin and stored at $4^{\circ} \mathrm{C}$ until further use.

In the treatment model (Figure 3A), mice received mammary fat pad injections of $4 \mathrm{~T} 1$ cells, were randomized the following day ( 24 hours after tumor injection) to receive PTH or PBS, and were followed for 4 weeks. In a separate experiment, mice were treated with either anti-mouse VCAM-1-neutralizing antibody (clone 429, MVCAM.A, Biolegend) or the matching isotype control IgG (Biolegend). Briefly, mice received mammary fat pad injections of $4 \mathrm{~T} 1$ cells and were randomized 24 hours later to receive PBS/IgG (control group), PTH ( $80 \mu \mathrm{g} / \mathrm{kg}$ /mouse Monday-Friday), or anti-VCAM-1 antibody (200 $\mu$ g twice a week followed by $80 \mu \mathrm{g} /$ mouse Monday-Friday for 3 weeks), as described in Figure 8E. After 4 weeks, animals were euthanized and individual organs were dissected and fixed in $10 \%$ formalin and stored at $4{ }^{\circ} \mathrm{C}$ until further use.

In the survival model, mice received mammary fat pad injections of 4T1 cells. All macroscopically visible primary tumors were surgically removed approximately 3 weeks (Figure 4A) or 2 weeks (Figure 4C) after $4 \mathrm{~T} 1$ injections, and the wounds were closed using nylon sutures. The mice received $5 \mathrm{mg} / \mathrm{kg}$ carprofen for analgesia. 24 hours after surgery mice were randomized to receive PTH or PBS and were monitored daily for survival. Following the Administrative Panels on Laboratory Animal Care guidelines for end point euthanasia, survival was defined using certain specific criteria: reappearance of primary tumor with any degree of ulceration, tumor size sufficient to interfere with normal activity or equal to $10 \%$ of preinoculation body weight, and/or moribund status. Mice were also monitored closely for clinical signs, including (but not limited to) rapid weight loss, abnormal respiratory rate/pattern, piloerection (ruffled coat), and listlessness. Treatment with PTH was continued until death or euthanasia due metastatic disease. Tumor and lungs were collected when possible for histological evaluation.

Intratibial injections. The pretreatment model approach was used for the intratibial injections, as shown in Figure 5A. 4T1 murine breast cancer cells were injected into syngeneic BALB/c mice and MDA human breast cancer cells were injected into athymic nude mice. Six-week-old mice were pretreated with either PTH [PTH(1-34); $80 \mu \mathrm{g} / \mathrm{kg} / \mathrm{d}]$ or PBS for 4 weeks prior to intratibial injection. 4T1 or MDA breast cancer cells grown to $70 \%$ confluence were harvested during the log phase of growth, and $1 \times 10^{3}$ cells were resuspended in $20 \mu 1$ of sterile PBS. The tumor cell suspensions were then injected into the right tibiae of the mice using a 27-gauge needle under isoflurane anesthesia. A slow drilling motion was used to advance the needle to avoid fractures. The left tibia was injected with the same volume of PBS (sham injected) to control for tumor cell injections. Treatments were continued for the next 4 weeks or until a 10\% difference in measurement between the two hind limbs was observed. Hind limb thickness was measured weekly using digital calipers. Hind limbs were fixed in $10 \%$ formalin and processed for $\mu \mathrm{CT}$ analyses and immunohistochemistry.

Calcein labeling. Double labeling of bones was performed with intraperitoneal injections of calcein (20 $\mathrm{mg} / \mathrm{kg}) 10$ and 3 days before euthanasia. The hind limbs were fixed, dehydrated, and methylmethacrylate resin embedded, and the calcein label was detected using florescence microscopy (75). 
A

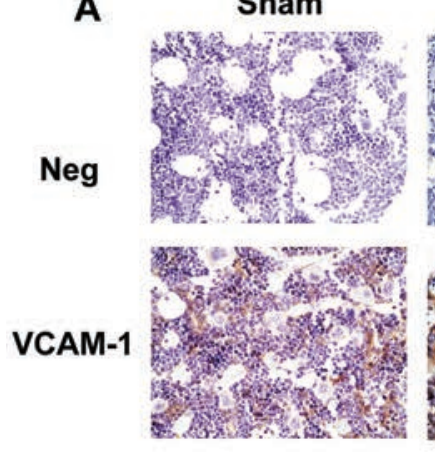

PBS
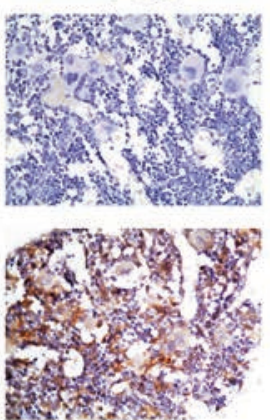

PTH

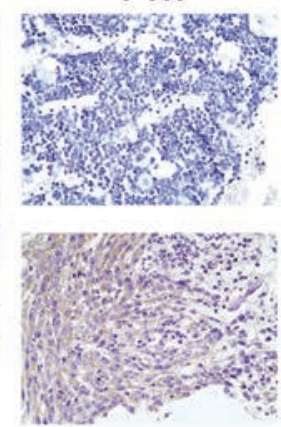

B

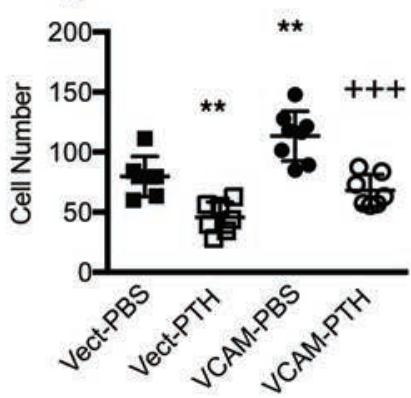

C

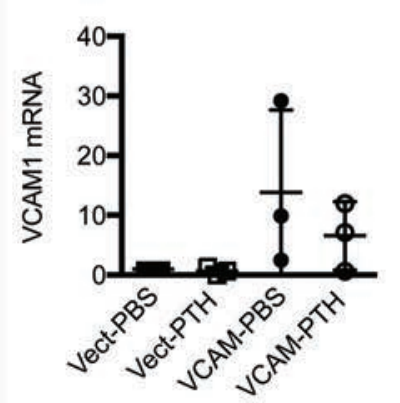

D

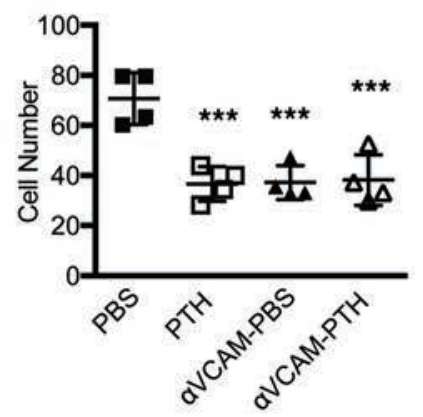

E

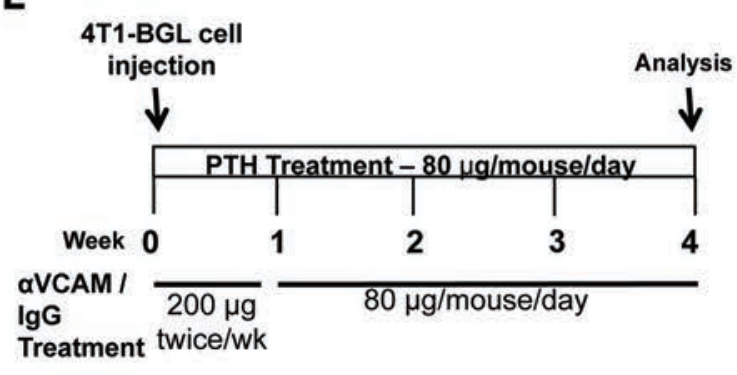

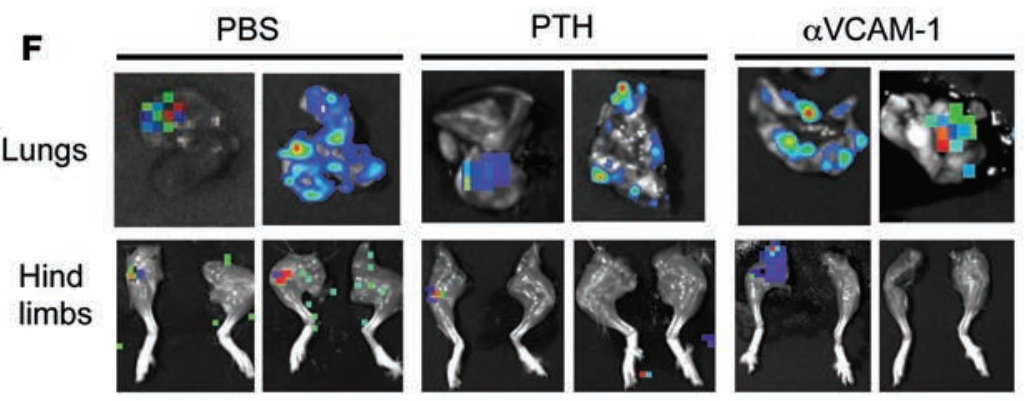

Figure 8. Decrease in VCAM-1 expression reduces the migratory potential of $4 \mathrm{T1}$ breast cancer cells both in vitro and in vivo. (A) Representative VCAM-1 staining in sham-, PBS-, and PTH-treated tibiae with 4 T1 cells (original magnification, $\times 40)(n=6)$. (B) Numbers of 4 T1 cells that migrated towards MC3T3-E1 cells overexpressing VCAM-1 treated with intermittent PTH or PBS in Transwell assays $(n=8)$. (C) Vcam1 mRNA levels in MC3T3-E1 cells overexpressing VCAM-1 and treated with intermittent PTH or PBS. (D) Numbers of 4T1 cells that migrated towards MC3T3-E1 cells treated with VCAM-1-neutralizing antibody $(20 \mu \mathrm{g} / \mathrm{ml})$ and intermittent PTH in Transwell assays $(n=5)$. (E) In vivo treatment with anti-VCAM-1 antibody experimental design $(n=10)$. (F) Representative BLI images of metastases to lungs and hind limbs. All values represent mean \pm SD of at least 3 individual experiments conducted in triplicate. ${ }^{* *} P<0.01,{ }^{* *} P<0.001$ when compared with PBS group. ${ }^{+++} P<0.001$ when compared with VCAM-PBS group, by 1-way ANOVA with Bonferroni's test as post-hoc analysis.

BLI. BLI was performed weekly on live animals under isoflurane anesthesia using an IVIS 200 Imaging platform (Caliper Life Sciences Inc.) following injection of luciferin substrate ( $3.33 \mathrm{mg} / \mathrm{mouse}$ ). Luciferin substrate was injected into the mice 10 minutes prior to euthanasia. Average radiance was measured and quantified for all organs using Living Image Software version 4.3.1. After BLI imaging, tumors were snap frozen and stored at $-80^{\circ} \mathrm{C}$ for RNA isolation. For the intratibial studies, BLI imaging was measured weekly using the AMI-X Imaging system (Spectral Instruments Imaging), which has X-ray as well as BLI measuring capabilities, and average radiance was quantitated using the AMIView software.

$\mu C T$ analyses. Tibiae dissected from mice bearing the $4 \mathrm{~T} 1$ intratibial tumors were scanned with a high-resolution microtomographic system (Scanco VicaCT 40, Scanco Medical AG). Tibiae were aligned perpendicular to the scanning axis, and the entire tibia was scanned. Scanning was conducted using the following settings: $55 \mathrm{kVp}$ and $145 \mu \mathrm{A}$ intensity, $200 \mathrm{~ms}$ integration time, and 1,000 projections, with a $0.5-\mathrm{mm} \mathrm{AI}$ filter at a resolution of $10 \mu \mathrm{m} /$ voxel. Each region of interest consisted of 
Table 4. Numbers of metastases to lungs, liver, spleen, and hind limbs in mice treated with PBS, PTH, or anti-VCAM-1 antibody

\begin{tabular}{|c|c|c|c|}
\hline \multirow{2}{*}{$\begin{array}{l}\text { Model } \\
\text { Group }\end{array}$} & \multicolumn{3}{|c|}{ Treatment } \\
\hline & PBS & PTH & $\alpha$ VCAM-1 \\
\hline Lungs & $7 / 10$ & $6 / 10$ & $7 / 10$ \\
\hline Liver & $1 / 10$ & $0 / 10$ & $1 / 10$ \\
\hline Spleen & $0 / 10$ & $0 / 10$ & $1 / 10$ \\
\hline Hind limbs & $7 / 10$ & $4 / 10$ & $4 / 10$ \\
\hline
\end{tabular}

approximately $100 \mu \mathrm{CT}$ sections, beginning $5 \%$ distal to the tibial growth plate and including the tibial metaphysis. 3D structural analyses were completed using the accompanying software.

Immunohistochemistry. Primary tumors, lungs, livers, spleens, and tibiae (cleaned from muscle and connective tissue) were harvested from treated and control mice and fixed in $10 \%$ neutral buffered formalin for $24-48$ hours at $4^{\circ} \mathrm{C}$, transferred to $70 \%$ ethanol, and stored until further use. The organs were macroscopically examined before fixation for presence of metastatic foci. The fixed tibiae were decalcified in $20 \%$ EDTA and processed along with the other organs for paraffin embedding. 5- $\mu \mathrm{M}$ sections of the paraffin-embedded tissue were then processed for H\&E staining to visualize pathology. Tumor burden in bone sections was defined by regions of interest, beginning $200 \mu \mathrm{m}$ distal to the tibial growth plate and extending 900 $\mu \mathrm{m}$ below and contained within the cortices. This was considered the total area. Tumor burden was traced within this same region of interest, and the final measurements were presented as percentage tumor burden of the total area using the ImageJ, version 1.48 (NIH). Immunostaining for expression of VCAM-1 (rabbit anti-VCAM-1 antibody EPR5047, Abcam) and CXCR7 (anti-human/mouse CXCR7 331111, Biolegend) was carried after overnight incubation with the primary antibody. The MACH 4 Universal HRP-Polymer Detection System (BRI4012, Biocare Medical) was used to detect peroxidase staining according to the manufacturer's instructions. Sections were also processed for TRAP staining using the leukocyte acid phosphatase kit (387A-1KT, Sigma-Aldrich) according to the manufacturer's protocol.

Flow cytometry. To determine the percentage of $\mathrm{GFP}^{+} 4 \mathrm{~T} 1$ cells in bone and bone marrow of tumor-bearing mice treated with PTH or PBS, femurs and tibiae were flushed of bone marrow and digested with $2.5 \%$ collagenase I and II (1:3 ratio). Bone marrow cells were simultaneously digested with $0.1 \%$ Collagenase IV (Gibco) and $0.2 \%$ Dispase (Sigma-Aldrich) at $37^{\circ} \mathrm{C}$ for 30 minutes. Single-cell suspensions were stained with anti-CD45-Biotin, anti-Ter119-Biotin, and anti-CD31-Biotin (Biolegend) to exclude hematopoietic cells and then stained with streptavidin-APC/Cy7 (Biolegend). Cells were resuspended in staining medium containing propidium iodide and analyzed on LSR II flow cytometer (BD Biosciences).

\section{Measurement of serum calcium and $1,25(\mathrm{OH})_{2}$ vitamin $\mathrm{D}$}

Serum calcium levels were measured using the QuantiChrom calcium assay kit (BioAssay Systems) following the manufacturer's protocol. Serum 1,25 (OH)D vitamin D levels were measured at Heartland Assays. The assay did not distinguish between vitamin $\mathrm{D}_{2}$ or $\mathrm{D}_{3}$ metabolites.

\section{Migration assays}

$2 \times 10^{4}$ MC3T3-E1 mouse preosteoblastic cells (ATCC) were cultured in aMEM media supplemented with $10 \% \mathrm{FBS}, 100 \mathrm{IU} / \mathrm{ml}$ penicillin, $100 \mu \mathrm{g} / \mathrm{ml}$ streptomycin, and then seeded in the lower chamber of a $8-\mu \mathrm{m}$ pore polycarbonate Transwell Permeable Support (Costar) system and allowed to attach overnight. Cells were then treated with either PBS (control) or PTH $(50 \mathrm{ng} / \mathrm{ml})$ and incubated for 6 hours. At the end of 6 hours, PTH-treated cells were divided into two groups. One group received fresh medium and PTH (continuous), while the other group received fresh medium without PTH (intermittent). The control group received fresh medium and PBS. Simultaneously, $5 \times 10^{4}$ breast cancer cells (4T1 or MDA) were seeded in the upper inset of the Transwell setup in serum-free medium. Cells in both chambers were incubated separately overnight at $37^{\circ} \mathrm{C}$ with $5 \% \mathrm{CO}_{2}$ in a humidified incubator. After overnight incubation the upper chamber was transferred to the Transwell setup and cells were allowed to migrate for 4 hours. At the end of 4 hours, cells in the upper chamber were wiped off. Cells that migrated to the under side of the membrane were fixed and stained with DAPI. Five random fields 


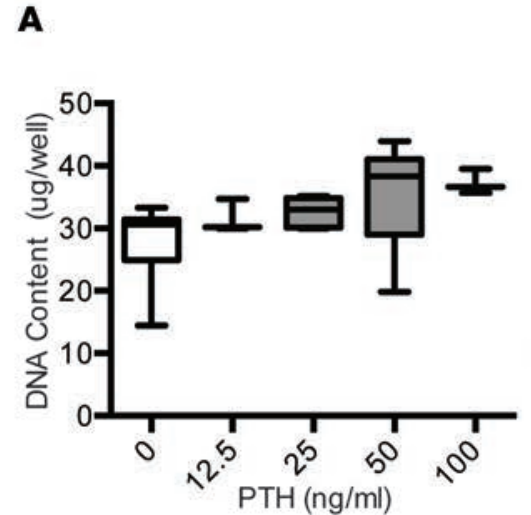

C

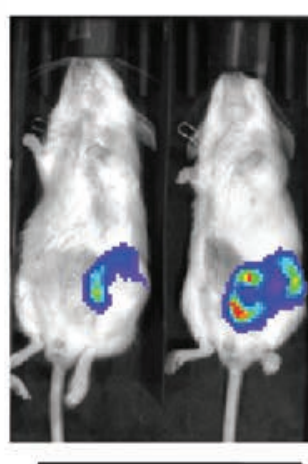

PBS

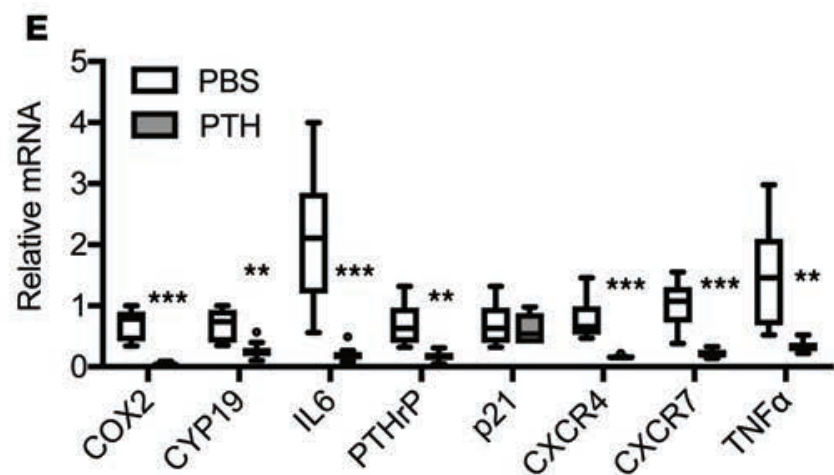

D
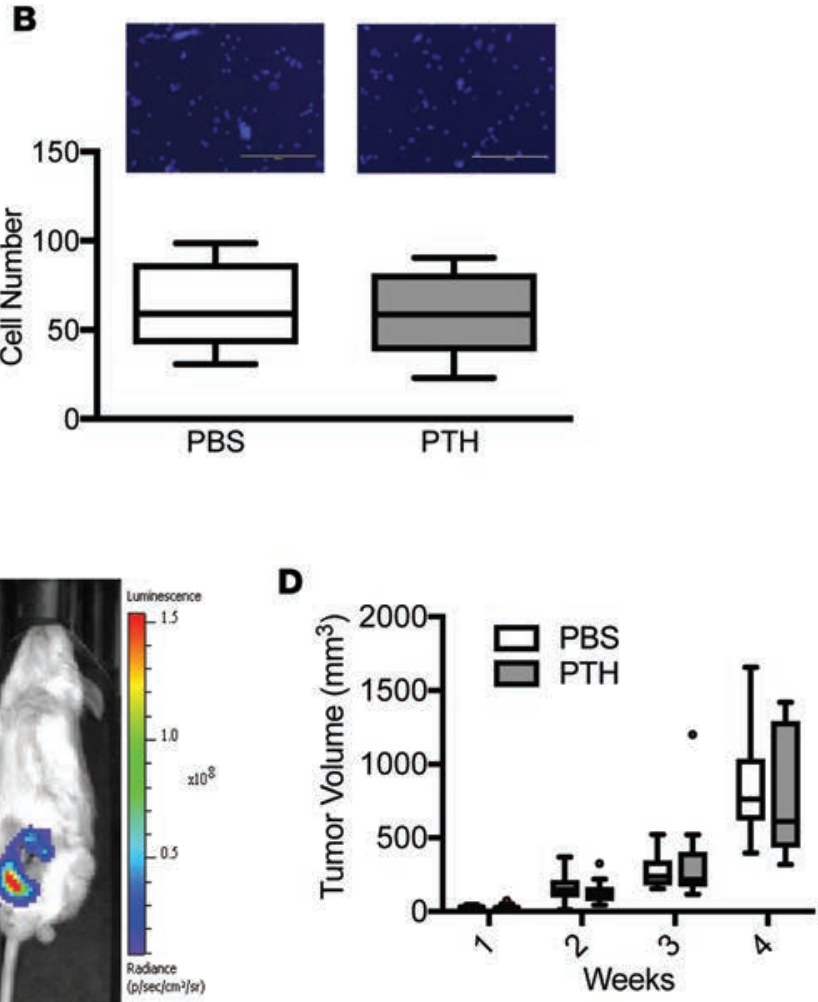

F

NEG

CXCR-7

PBS
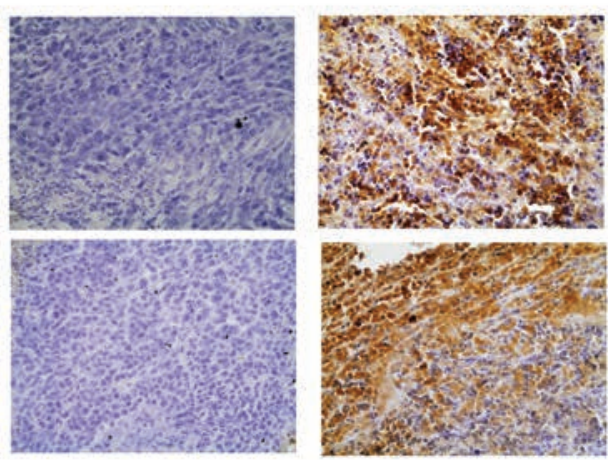

Figure 9. PTH administration does not affect 4T1 cell growth in vitro or tumor progression in BALB/c mice. (A) DNA content in 4T1 cells treated with increasing concentrations of intermittent PTH for 6 days. (B) 4T1 cells were treated with intermittent PTH ( 6 of 24 hours) and allowed to migrate against a serum gradient in a Transwell assay. All values represent mean \pm SD of at least 3 individual experiments conducted in triplicate. Scale bar: $200 \mu \mathrm{m}$. (C) Representative BLI at end point and (D) weekly tumor volume in mice injected with 4T1 cells and treated with PTH (treatment model) for 4 weeks. (E) Expression of select target genes in primary tumors dissected from mice pretreated with PTH, as described in Figure 1A. (F) Representative CXCR7 staining in primary tumors treated with PTH, as described in Figure 1A (original magnification, $\times 40$ ). All values represent mean \pm SD $(n=10)$ for each group. ${ }^{* *} P<$ $0.01,{ }^{* *} P<0.001$ when compared with PBS group, by 1-way ANOVA with Bonferroni's test as post-hoc analysis.

per membrane were photographed using a fluorescence microscope and total numbers of cells were counted using the ImageJ, version 1.48 (NIH). In a separate experiment, condition media was collected after intermittent PTH treatment and used to assess migration potential of 4T1 cells in the absence of MC3T3-E1 cells. To test the direct effects of PTH on breast cancer cells, 4T1 cells were treated with either PBS or PTH $(50 \mathrm{ng} / \mathrm{ml})$ for 6 hours as described above. After overnight incubation $1 \times 10^{5}$ cells were seeded in the upper inset of the Transwell chamber, allowed to migrate against a serum gradient for 6 hours, and processed as described above. 


\section{Overexpression of VCAM-1}

Mouse CD106/VCAM-1 natural ORF mammalian expression plasmid in a pCMV3 vector was obtained from Sino Biological Inc. MC3T3-E1 cells were seeded in the lower chamber of the Transwell migration setup as described above. Transient transfections were then performed to overexpress VCAM-1 using Lipofectamine (Invitrogen) for 24 hours according to the manufacturer's instructions. RNA was isolated and expression of Vcam1 was confirmed using gene-specific primers provided by the manufacturer (Sino Biological Inc.). Empty vectors were used as negative controls for the expression of VCAM-1. MC3T3-E1 cells transiently transfected with VCAM-1 were treated with either PBS or PTH, and migrations of 4T1 cells were assessed as described in the previous section. Overexpression of VCAM-1 in the transfected cells was assessed by real-time PCR using gene-specific primers provided by the manufacturer. In a separate experiment, MC3T3-E1 cells were treated with VCAM-1-neutralizing antibody $(20 \mu \mathrm{g} / \mathrm{ml})$ (LEAF-purified anti-mouse CD106 antibody, Biolegend) in the presence or absence of intermittent PTH treatment, and migrations were assessed as described earlier.

\section{RNA isolation and real-time PCR}

Total RNA was isolated from MC3T3-E1 cells and primary tumors following homogenization using the Trizol reagent (Invitrogen) according to the manufacturer's instructions. $5 \mu \mathrm{g}$ RNA was subjected to reverse transcription using the SuperScript III first strand synthesis kit (Invitrogen), and gene expression was determined by real-time PCR using the CFX96 real-time PCR detection system (Bio-Rad), with gene-specific primers (Supplemental Table 2) and SYBR green qPCR kit (Bio-Rad). Target gene expressions were normalized to $\beta$-actin, and the relative changes in mRNA levels were assessed by the comparative $C_{T}$ method (76). To determine the effects of intermittent PTH on the genes involved in tumor metastasis, RNA was isolated from MC3T3-E1 cells treated with intermittent PTH and cocultured with 4T1 cells, and expression levels of 88 tumor metastasis genes were determined using the $\mathrm{RT}^{2}$ Profiler Tumor Metastasis PCR Array (Qiagen) according to the manufacturer's instructions. Gene-specific primers were used to validate expression of genes that were significantly changed.

\section{CXCL-12 ELISA}

MC3T3-E1 cells plated in Transwell migration chambers were treated with PBS/PTH as described above and incubated in the presence or absence of $4 \mathrm{~T} 1$ cells for 4 hours. The cells and media were collected for the analysis of CXCL-12/SDF-1 using a quantikine ELISA kit (R\&D Systems) according to the manufacturer's instructions.

\section{Cell proliferation assay}

$4 \mathrm{~T} 1$ cells were seeded (100,000 cells/well) in 6-well culture plates in DMEM/F12 medium containing $10 \%$ FBS and treated with graded concentrations of PTH as described earlier (77). Cell proliferation was assessed at the end of 6 days by measuring total DNA content, as described earlier (78).

\section{Statistics}

Statistical analyses were performed using GraphPad Prism 5 software (GraphPad Software). $Z$ proportion scores were calculated where applicable, and data were evaluated using ANOVA with multiple comparisons or 1-way ANOVA with Bonferroni's test as the post-hoc analysis. $P$ values of less than 0.05 were considered significant.

\section{Study approval}

All animal procedures were performed in compliance with the guidelines approved by the Stanford University Administrative Panels on Laboratory Animal Care.

\section{Author contributions}

Conception and design were provided by JYW, SS, and RWJ. Development of methodology was performed by SS, JJ, LAB, and MAA. Acquisition of data was performed by SS, JJ, LAB, TK, and HZ. Analysis and interpretation of data were performed by SS and JYW. Writing, review, and revision of the manuscript were performed by SS, JYW, and RWJ. Administrative, technical, and material support was provided by SS, JYW, and RWJ. Study supervision was provided by JYW and SS. 


\section{Acknowledgments}

This study was supported in part with funding from the Mary Kay Foundation (to JYW), the NIH (OD008466 to JYW and R00CA194198 to RWJ), and the Department of Defense (W81XWH-17-1-0027 to JYW).

Address correspondence to: Joy Y. Wu, Stanford University School of Medicine, 300 Pasteur Drive, Room S025, Stanford California 94305-5103, USA. Phone: 650.736.9654; Email: jywu1@stanford.edu.

1. Siegel RL, Miller KD, Jemal A. Cancer statistics, 2015. CA Cancer J Clin. 2015;65(1):5-29.

2. Eckhardt BL, Francis PA, Parker BS, Anderson RL. Strategies for the discovery and development of therapies for metastatic breast cancer. Nat Rev Drug Discov. 2012;11 (6):479-497.

3. Coleman RE. Clinical features of metastatic bone disease and risk of skeletal morbidity. Clin Cancer Res. 2006;12 (20 Pt 2):6243s-6249s.

4. Kiel MJ, Morrison SJ. Uncertainty in the niches that maintain haematopoietic stem cells. Nat Rev Immunol. 2008;8 (4):290-301.

5. Nakamura $Y$, et al. Isolation and characterization of endosteal niche cell populations that regulate hematopoietic stem cells. Blood. 2010;116 (9):1422-1432.

6. Shiozawa Y, Havens AM, Pienta KJ, Taichman RS. The bone marrow niche: habitat to hematopoietic and mesenchymal stem cells, and unwitting host to molecular parasites. Leukemia. 2008;22 (5):941-950.

7. Sinha P, et al. Loss of Gs $\alpha$ early in the osteoblast lineage favors adipogenic differentiation of mesenchymal progenitors and committed osteoblast precursors. J Bone Miner Res. 2014;29 (11):2414-2426.

8. Taichman RS. Blood and bone: two tissues whose fates are intertwined to create the hematopoietic stem-cell niche. Blood. 2005;105 (7):2631-2639.

9. Wu JY, et al. Osteoblastic regulation of B lymphopoiesis is mediated by Gs $\{a l p h a\}$-dependent signaling pathways. Proc Natl Acad Sci USA. 2008;105 (44):16976-16981.

10. Wu JY, Scadden DT, Kronenberg HM. Role of the osteoblast lineage in the bone marrow hematopoietic niches. J Bone Miner Res. 2009;24 (5):759-764.

11. Yin T, Li L. The stem cell niches in bone. J Clin Invest. 2006;116(5):1195-1201.

12. Dallas SL, Rosser JL, Mundy GR, Bonewald LF. Proteolysis of latent transforming growth factor-beta (TGF-beta )-binding protein-1 by osteoclasts. A cellular mechanism for release of TGF-beta from bone matrix. J Biol Chem. 2002;277 (24):21352-21360.

13. Luker KE, Luker GD. Functions of CXCL12 and CXCR4 in breast cancer. Cancer Lett. 2006;238 (1):30-41.

14. Schneider JG, Amend SR, Weilbaecher KN. Integrins and bone metastasis: integrating tumor cell and stromal cell interactions. Bone. 2011;48 (1):54-65.

15. Sethi N, Dai X, Winter CG, Kang Y. Tumor-derived JAGGED1 promotes osteolytic bone metastasis of breast cancer by engaging notch signaling in bone cells. Cancer Cell. 2011;19 (2):192-205.

16. Shapiro CL, Manola J, Leboff M. Ovarian failure after adjuvant chemotherapy is associated with rapid bone loss in women with early-stage breast cancer. J Clin Oncol. 2001;19 (14):3306-3311.

17. Cepa M, Vaz C. Management of bone loss in postmenopausal breast cancer patients treated with aromatase inhibitors. Acta Reumatol Port. 2015;40 (4):323-330.

18. Bjarnason NH, Hitz M, Jorgensen NR, Vestergaard P. Adverse bone effects during pharmacological breast cancer therapy. Acta Oncol. 2008;47 (4):747-754.

19. Sun L, Yu S. Efficacy and safety of denosumab versus zoledronic acid in patients with bone metastases: a systematic review and meta-analysis. Am J Clin Oncol. 2013;36 (4):399-403.

20. Stopeck AT, et al. Denosumab compared with zoledronic acid for the treatment of bone metastases in patients with advanced breast cancer: a randomized, double-blind study. J Clin Oncol. 2010;28 (35):5132-5139.

21. Rollason V, Laverrière A, MacDonald LC, Walsh T, Tramèr MR, Vogt-Ferrier NB. Interventions for treating bisphosphonate-related osteonecrosis of the jaw (BRONJ). Cochrane Database Syst Rev. 2016;2:CD008455.

22. Ruggiero SL, et al. American Association of Oral and Maxillofacial Surgeons position paper on bisphosphonate-related osteonecrosis of the jaws--2009 update. J Oral Maxillofac Surg. 2009;67(5 Suppl):2-12.

23. Ruggiero SL, et al. American Association of Oral and Maxillofacial Surgeons position paper on medication-related osteonecrosis of the jaw--2014 update. J Oral Maxillofac Surg. 2014;72(10):1938-1956.

24. Yaccoby S, et al. Inhibitory effects of osteoblasts and increased bone formation on myeloma in novel culture systems and a myelomatous mouse model. Haematologica. 2006;91 (2):192-199.

25. Krawetz R, Wu YE, Rancourt DE, Matyas J. Osteoblasts suppress high bone turnover caused by osteolytic breast cancer in-vitro. Exp Cell Res. 2009;315 (14):2333-2342.

26. Phadke PA, et al. Kinetics of metastatic breast cancer cell trafficking in bone. Clin Cancer Res. 2006;12 (5):1431-1440.

27. Fitzpatrick LA, Bilezikian JP, Silverberg SJ. Actions of parathyroid hormone on the vasculature and cardiovascular system. In: Bilezikian JP, Raisz LG, Martin TJ, eds. Principles of Bone Biology. San Diego, CA, USA: Academic Press Inc.; 2008:657-664.

28. Miller PD, et al. Effect of abaloparatide vs placebo on new vertebral fractures in postmenopausal women with osteoporosis: a randomized clinical trial. JAMA. 2016;316 (7):722-733.

29. Neer RM, et al. Effect of parathyroid hormone (1-34) on fractures and bone mineral density in postmenopausal women with osteoporosis. N Engl J Med. 2001;344 (19):1434-1441.

30. Horwitz MJ, et al. A 7-day continuous infusion of PTH or PTHrP suppresses bone formation and uncouples bone turnover. $J$ Bone Miner Res. 2011;26(9):2287-2297.

31. Deal C, Gideon J. Recombinant human PTH 1-34 (Forteo): an anabolic drug for osteoporosis. Cleve Clin J Med. 2003;70(7):5856,589 . 
32. Adams GB, et al. Therapeutic targeting of a stem cell niche. Nat Biotechnol. 2007;25(2):238-243.

33. Calvi LM, et al. Osteoblastic cells regulate the haematopoietic stem cell niche. Nature. 2003;425 (6960):841-846

34. Yu EW, et al. Teriparatide (PTH 1-34) treatment increases peripheral hematopoietic stem cells in postmenopausal women. $J$ Bone Miner Res. 2014;29 (6):1380-1386.

35. Pennisi A, et al. Consequences of daily administered parathyroid hormone on myeloma growth, bone disease, and molecular profiling of whole myelomatous bone. PLoS One. 2010;5(12):e15233.

36. Krause DS, et al. Differential regulation of myeloid leukemias by the bone marrow microenvironment. Nat Med. 2013;19 (11):1513-1517

37. Fontanella C, Fanotto V, Rihawi K, Aprile G, Puglisi F. Skeletal metastases from breast cancer: pathogenesis of bone tropism and treatment strategy. Clin Exp Metastasis. 2015;32 (8):819-833.

38. Yamada Y, et al. Plasma concentrations of VCAM-1 and PAI-1: a predictive biomarker for post-operative recurrence in colorectal cancer. Cancer Sci. 2010;101 (8):1886-1890.

39. Holubec L, et al. Markers of cellular adhesion in diagnosis and therapy control of colorectal carcinoma. Anticancer Res. 2005;25(3A):1597-1601

40. Yurkovetsky Z, et al. Development of a multimarker assay for early detection of ovarian cancer. J Clin Oncol. 2010;28 (13):21592166.

41. Panaroni C, Fulzele K, Saini V, Chubb R, Pajevic PD, Wu JY. PTH Signaling in osteoprogenitors Is essential for B-lymphocyte differentiation and mobilization. J Bone Miner Res. 2015;30 (12):2273-2286.

42. Brown JE, Coleman RE. Denosumab in patients with cancer-a surgical strike against the osteoclast. Nat Rev Clin Oncol. 2012;9 (2):110-118.

43. Gnant M, Clézardin P. Direct and indirect anticancer activity of bisphosphonates: a brief review of published literature. Cancer Treat Rev. 2012;38 (5):407-415.

44. Jolette J, et al. Defining a noncarcinogenic dose of recombinant human parathyroid hormone 1-84 in a 2-year study in Fischer 344 rats. Toxicol Pathol. 2006;34 (7):929-940.

45. Vahle JL, Long GG, Sandusky G, Westmore M, Ma YL, Sato M. Bone neoplasms in F344 rats given teriparatide [rhPTH(1-34)] are dependent on duration of treatment and dose. Toxicol Pathol. 2004;32(4):426-438.

46. Vahle JL, et al. Skeletal changes in rats given daily subcutaneous injections of recombinant human parathyroid hormone (1-34) for 2 years and relevance to human safety. Toxicol Pathol. 2002;30 (3):312-321.

47. Elraiyah T, Gionfriddo MR, Murad MH. Acting on black box warnings requires a GRADE evidence table and an implementation guide: the case of teriparatide. J Clin Epidemiol. 2015;68 (6):698-702.

48. Cipriani C, Capriani C, Irani D, Bilezikian JP. Safety of osteoanabolic therapy: a decade of experience. J Bone Miner Res. 2012;27 (12):2419-2428.

49. Osagie-Clouard L, Sanghani A, Coathup M, Briggs T, Bostrom M, Blunn G. Parathyroid hormone 1-34 and skeletal anabolic action: The use of parathyroid hormone in bone formation. Bone Joint Res. 2017;6 (1):14-21.

50. Silva BC, Bilezikian JP. Parathyroid hormone: anabolic and catabolic actions on the skeleton. Curr Opin Pharmacol. 2015;22:41-50.

51. Aslan D, et al. Mechanisms for the bone anabolic effect of parathyroid hormone treatment in humans. Scand J Clin Lab Invest. 2012;72 (1):14-22.

52. O’Brien CA, Nakashima T, Takayanagi H. Osteocyte control of osteoclastogenesis. Bone. 2013;54 (2):258-263.

53. Onyia JE, et al. Molecular profile of catabolic versus anabolic treatment regimens of parathyroid hormone (PTH) in rat bone: an analysis by DNA microarray. J Cell Biochem. 2005;95 (2):403-418.

54. Li X, et al. Determination of dual effects of parathyroid hormone on skeletal gene expression in vivo by microarray and network analysis. J Biol Chem. 2007;282 (45):33086-33097.

55. Bilezikian JP. Approaches to parathyroid disorders. In Rosen CJ, ed. Primer on the metabolic bone diseases and disorders of mineral metabolism. Ames, Iowa, USA: Wiley-Blackwell; 2013:537-542.

56. Michels KB, Xue F, Brandt L, Ekbom A. Hyperparathyroidism and subsequent incidence of breast cancer. Int J Cancer. 2004;110 (3):449-451.

57. Nilsson IL, Zedenius J, Yin L, Ekbom A. The association between primary hyperparathyroidism and malignancy: nationwide cohort analysis on cancer incidence after parathyroidectomy. Endocr Relat Cancer. 2007;14 (1):135-140.

58. Fierabracci $\mathrm{P}$, et al. Increased prevalence of primary hyperparathyroidism in treated breast cancer. J Endocrinol Invest. 2001;24 (5):315-320.

59. Tanaka Y. Primary hyperparathyroidism with breast carcinoma. Breast Cancer. 2010;17(4):265-268.

60. Shiozawa Y, et al. Human prostate cancer metastases target the hematopoietic stem cell niche to establish footholds in mouse bone marrow. J Clin Invest. 2011;121 (4):1298-1312.

61. Schneider A, et al. Bone turnover mediates preferential localization of prostate cancer in the skeleton. Endocrinology. 2005;146 (4):1727-1736.

62. Barcellos-Hoff MH, Lyden D, Wang TC. The evolution of the cancer niche during multistage carcinogenesis. Nat Rev Cancer. 2013;13 (7):511-518.

63. Kaplan RN, Psaila B, Lyden D. Bone marrow cells in the 'pre-metastatic niche': within bone and beyond. Cancer Metastasis Rev. 2006;25(4):521-529.

64. Peinado H, Lavotshkin S, Lyden D. The secreted factors responsible for pre-metastatic niche formation: old sayings and new thoughts. Semin Cancer Biol. 2011;21 (2):139-146.

65. Ma YL, et al. Catabolic effects of continuous human PTH (1--38) in vivo is associated with sustained stimulation of RANKL and inhibition of osteoprotegerin and gene-associated bone formation. Endocrinology. 2001;142 (9):4047-4054.

66. Qin L, Raggatt LJ, Partridge NC. Parathyroid hormone: a double-edged sword for bone metabolism. Trends Endocrinol Metab. 2004; 15 (2):60-65

67. Soki FN, Park SI, McCauley LK. The multifaceted actions of PTHrP in skeletal metastasis. Future Oncol. $2012 ; 8$ (7):803-817.

68. Zheng L, et al. PTHrP expression in human MDA-MB-231 breast cancer cells is critical for tumor growth and survival and osteoblast inhibition. Int J Biol Sci. 2013;9 (8):830-841. 
69. Martin TJ. Parathyroid hormone-related protein, its regulation of cartilage and bone development, and role in treating bone diseases. Physiol Rev. 2016;96 (3):831-871.

70. Guise TA, et al. Evidence for a causal role of parathyroid hormone-related protein in the pathogenesis of human breast cancer-mediated osteolysis. J Clin Invest. 1996;98 (7):1544-1549.

71. Yin JJ, et al. TGF-beta signaling blockade inhibits PTHrP secretion by breast cancer cells and bone metastases development. $J$ Clin Invest. 1999;103 (2):197-206.

72. Contag $\mathrm{CH}$, et al. Monitoring dynamic interactions between breast cancer cells and human bone tissue in a co-culture model. Mol Imaging Biol. 2014;16 (2):158-166.

73. Multhaup M, et al. Cytotoxicity associated with artemis overexpression after lentiviral vector-mediated gene transfer. Hum Gene Ther. 2010;21 (7):865-875.

74. Klein KA, et al. Progression of metastatic human prostate cancer to androgen independence in immunodeficient SCID mice. Nat Med. 1997;3 (4):402-408.

75. Sims NA, et al. Bone homeostasis in growth hormone receptor-null mice is restored by IGF-I but independent of Stat5. J Clin Invest. 2000;106 (9):1095-1103.

76. Livak KJ, Schmittgen TD. Analysis of relative gene expression data using real-time quantitative PCR and the 2 (-Delta Delta C (T)) Method. Methods. 2001;25 (4):402-408.

77. Ishizuya T, et al. Parathyroid hormone exerts disparate effects on osteoblast differentiation depending on exposure time in rat osteoblastic cells. J Clin Invest. 1997;99 (12):2961-2970.

78. Swami S, Krishnan AV, Feldman D. 1alpha,25-Dihydroxyvitamin D3 down-regulates estrogen receptor abundance and suppresses estrogen actions in MCF-7 human breast cancer cells. Clin Cancer Res. 2000;6 (8):3371-3379. 